\title{
A neuropsychosocial signature predicts longitudinal symptom changes in women with irritable bowel syndrome
}

\author{
Ravi R. Bhatt $\mathbb{D}^{1,2}$, Arpana Gupta ${ }^{1}{ }^{1}$, Jennifer S. Labus ${ }^{1}$, Cathy Liu ${ }^{1}$, Priten P. Vora ${ }^{1}$, Jean Stains ${ }^{1}$, Bruce D. Naliboff ${ }^{1}$ and \\ Emeran A. Mayer (iD) ${ }^{1 \times}$ \\ (c) The Author(s) 2021
}

\begin{abstract}
Irritable bowel syndrome (IBS) is a common disorder of brain-gut interactions characterized by chronic abdominal pain, altered bowel movements, often accompanied by somatic and psychiatric comorbidities. We aimed to test the hypothesis that a baseline phenotype composed of multi-modal neuroimaging and clinical features predicts clinical improvement on the IBS Symptom Severity Scale (IBS-SSS) at 3 and 12 months without any targeted intervention. Female participants $(N=60)$ were identified as "improvers" (50-point decrease on IBS-SSS from baseline) or "non-improvers." Data integration analysis using latent components (DIABLO) was applied to a training and test dataset to determine whether a limited number of sets of multiple correlated baseline'omics data types, including brain morphometry, anatomical connectivity, resting-state functional connectivity, and clinical features could accurately predict improver status. The derived predictive models predicted improvement status at 3-months and 12-months with $91 \%$ and $83 \%$ accuracy, respectively. Across both time points, non-improvers were classified as having greater correlated morphometry, anatomical connectivity and resting-state functional connectivity characteristics within salience and sensorimotor networks associated with greater pain unpleasantness, but lower default mode network integrity and connectivity. This suggests that non-improvers have a greater engagement of attentional systems to perseverate on painful visceral stimuli, predicting IBS exacerbation. The ability of baseline multimodal brain-clinical signatures to predict symptom trajectories may have implications in guiding integrative treatment in the age of precision medicine, such as treatments targeted at changing attentional systems such as mindfulness or cognitive behavioral therapy.
\end{abstract}

Molecular Psychiatry (2022) 27:1774-1791; https://doi.org/10.1038/s41380-021-01375-9

\section{INTRODUCTION}

Irritable bowel syndrome (IBS) is characterized by altered brain-gut interactions, chronic abdominal pain, altered bowel movements, and on occasion, psychiatric symptoms, (e.g., anxiety) [1]. In 2012, an estimated $11.3 \%$ of the global population was diagnosed with IBS, $65 \%$ being female $[2,3]$. For a subset of patients, IBS results in debilitating symptoms and greatly impacts quality of life [4]. Effective management of IBS requires an integrative approach which may include a combination of educational, pharmacological, psychological, and behavioral treatments [5]. Identifying biomarkers to predict symptom improvement can assist specialists in the practice of precision medicine. Tailored and multifaceted treatments are critical when symptoms vary from patient to patient, such as with IBS and chronic pain [1, 6-8].

IBS pathophysiology has been informed by neuroimaging studies that reveal affected innate and elicited brain structure and activity. Brain networks involved in central processing and modulation of IBS-related visceral pain include the salience, sensorimotor, default mode, emotional arousal, central executive, and central autonomic networks $[9,10]$. Evidence shows dynamic interactions between brain networks are altered in IBS. These adaptations may influence key information processing systems in terms of attention, memory, perceptions, problem solving, and planning, as well as, autonomic hyperarousal [10]. Efferent signals from the periaqueductal gray and raphe nuclei play crucial roles in descending endogenous pain modulation via limbic and cortical input, regulating dorsal horn excitability in response to visceral afferent input [11]. Vagal and sympathetic efferent projections from the central autonomic network through the brainstem modulate the activity of the enteric nervous system (ENS), and afferents from the ENS send viscerosensory signals back to the brain. A variety of mechanisms contributing to IBS symptoms have been identified which are involved in brain-gut microbiome interactions, including the gut microbiome, metabolome, neuroimmune interactions, genetics, as well as psychological and behavioral history $[1,10,12]$.

Moreover, many psychosocial and environmental factors are known to play a large role in the susceptibility, development, symptom flares, and chronicity of IBS $[1,10]$. These include, but are not limited to, environmental aspects such as early life adversity [13-15], personality traits such as neuroticism [16, 17], coping strategies such as catastrophizing behavior [18, 19], and negative emotions and psychiatric disorders such as anxiety, depression, and somatoform disorders [1, 20,21]. Throughout life, all of these

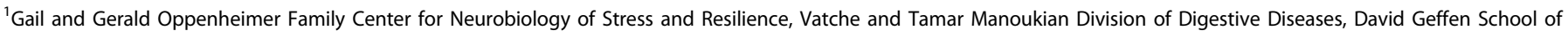

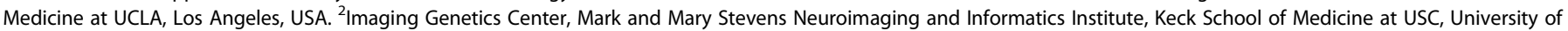
Southern California, Los Angeles, USA. ${ }^{凶}$ email: emayer@ucla.edu
}

Received: 11 February 2021 Revised: 19 October 2021 Accepted: 20 October 2021

Published online: 24 November 2021 
factors can influence dysregulation of the brain-gut axis through neural, neuroimmune, and neuroendocrine pathways which connect the brain and the gastrointestinal tract [1, 10]. Considering psychosocial factors when assessing IBS pathophysiology in addition to the biological factors underpinning IBS leads to more targeted and efficacious pharmacologic and non-pharmacologic treatments [1, 10, 12].

Based on the possibility of using neuroimaging and behavioral data to estimate disease trajectory in brain-gut interactions [12], identification of a signature from longitudinal data could help identify those with a more negative disease trajectory and responsiveness to treatment. In other chronic pain syndromes such as chronic pelvic pain [22] and low back pain [23-25], baseline neuroimaging data have been shown to predict symptom changes longitudinally, but to date no multi-modal brain and clinical phenotype has been identified which can predict symptom trajectories in IBS.

This observational cohort study reports the results of an ongoing deep phenotyping study (NCT02693730) in patients with IBS. We aimed to predict a baseline phenotype using patient data composed of multi-modal neuroimaging, behavioral testing, and clinical self-report questionnaires and hypothesized that an integrated neuropsychosocial signature is able to classify IBS patients into those that improve over time and those who do not. To test this hypothesis, we examined changes across these variables at 3 and 12 months by IBS symptom severity. We analyzed three whole-brain imaging modalities: structural morphometry, resting-state functional connectivity, and anatomical connectivity assessed by diffusion imaging and used a multi-omics integrative approach to identify distinct signatures [26]. Additionally, behavioral data and clinical questionnaires were used as an additional "-omic" type to understand how relationships between the brain and behavior could predict longitudinal outcomes. This signature makes it possible to connect specific psychosocial factors (including early life adversity, resilience, symptom severity and perceived pain), with whole-brain neurobiological patterns of morphometry, resting-state functional connectivity, and anatomical connectivity.

\section{METHODS \\ Participants}

Sixty female participants were recruited by the Center for Neurobiology of Stress and Resilience at UCLA starting in 2016 (mean age $=29.08$, SD $=$ 11.46). All met Rome III criteria for IBS, including subtypes of bowel habit predominance [27]. Those reporting histories of the following were excluded: other gastrointestinal illnesses; eating disorders; rectal prolapse; severe hemorrhoids; gastric, abdominal, or colon surgery; recent steroid use; insulin-dependent diabetes; kidney disease; heart disease; hypertension; cancer; significant lung disease and/or neurological condition (TBI, seizures); major surgery within 6 months of study onset, other chronic illness and/or pain condition; nicotine use; alcohol or drug misuse, psychiatric or developmental disorders impairing self-report, use of HIV and/or SSRI medications, recent clinical trial participation (i.e., 28 days), and variables obstructing MRI testing (e.g., metal implants, whole-limb tattoos, claustrophobia, untreated anxiety, or panic attacks). All participants received informed consent about the procedures of the study.

\section{Study design}

In this natural observation study, patients with IBS participated in multimodal brain imaging, quantitative sensory testing, and a broad range of psychosocial questionnaires at baseline, 3 and 12 months of follow-up. There were 122 patients screened for eligibility; 78 confirmed eligible; 76 decided to enroll in the study. The sample size dropped to 60 at 3 months, and to 43 at 12 months due to attrition. There were 21 subjects who dropped from the study across 12 months, resulting in 55 distinct subjects completing the study. As this analysis is part of a larger, ongoing deep phenotyping study, the full procedures are in the Supplementary Methods: Study Design. Briefly, the initial screening visit consisted of the following in order; informed consent, assessing eligibility, reviewing contaminant medications, taking vital signs, a medical history and exam, a psychological interview, a blood sample, thermal quantitative sensory testing (QST), a battery of psychosocial questionnaires, and finally a urine pregnancy test. The following visit (visit 2) was the first MRI visit and consisted of the following in order; contaminant medication conditions were reviewed, adverse events reviewed, a battery of psychosocial questionnaires, brain imaging procedures, a urine pregnancy test, and a stool sample. The following MRI visits at 3 and 12 months included the following in order; eligibility assessment, contaminant conditions review, weight and vital signs, blood sample, adverse events review, thermal QST, psychosocial questionnaires, brain imaging, a urine pregnancy test, and a stool sample.

\section{Behavioral data/Clinical questionnaires}

Behavioral and clinical variables taken at baseline are detailed in the "Supplementary Methods: Questionnaires". The IBS Symptom Severity Scale (IBS-SSS) measured patients' self-report of IBS symptom severity. Scores less than 75 indicate controls, between 75 and 175 indicate mild IBS, between 175 and 300 indicate moderate IBS, and greater than 300 indicates severe IBS. It has good reproducibility and is sensitive to change [28]. Over 12 months at 3-month intervals, each participant completed the IBS-SSS. IBS-SSS scores were used to separate participants into two distinct symptom groups. Patient symptoms were defined as "improving" if IBS-SSS scores were reduced by 50-points or more. For ease of discussion, this symptom group was labeled, "Improvers." Patient symptoms were otherwise defined as persistent or worsening (i.e., no change, increases, or decreases of less than 50-points in IBS-SSS score), and placed into the "Non-Improvers" group [28]. As hormonal status can also play a role in abdominal symptoms, psychological state, and pain perception [29], the use of hormonal contraceptives was also recorded $(N=15$, "Supplementary Methods: Hormonal Status and use of Contraceptives" Table S1). Fiftyfive women were premenopausal, one woman was perimenopausal, and four women were postmenopausal.

Other self-report variables used in our analyses included the Bowel Symptom Questionnaire (BSQ) [30], Hospital Anxiety and Depression Scale (HADS) [31], State-Trait Anxiety Inventory (STAI) [32], Perceived Stress Scale (PSS) [33], International Personality Item Pool (IPIP) [34], Early Trauma Inventory (ETI) [35], Complex Multi-Symptom Inventory (CMSI) [36], and the Connor-Davidson Resilience Scale (CD-RISC) [37].

\section{Behavioral data}

Quantitative sensory testing (QST) at baseline involved a brief thermal pain sensitivity test using an electrically heated circular thermode placed on the forearm. Thermode surface area was $3 \mathrm{~cm} \times 3 \mathrm{~cm}$. Testing consisted of six randomized trials. The thermode temperature increased from $32^{\circ} \mathrm{C}$ by $0.5^{\circ} \mathrm{C} / \mathrm{s}$ with each trial, and returned to $32^{\circ} \mathrm{C}$ by $10^{\circ} \mathrm{C} / \mathrm{s}$. In three trials, participants spoke when pain was perceived. This was recorded as "pain threshold". In the other three trials, participants spoke when pain was intolerable. This was recorded as "pain tolerance". A delay of $20 \mathrm{~s}$ occurred between trials. The thermode placement was slightly altered between each trial to avoid the sensitization or habituation of cutaneous receptors [38]. Pain threshold and tolerance scores were averaged to compute final scores. Pain intensity and unpleasantness of the tasks were then rated on a 0-20 visual analog scale using the Gracely Pain Scale [39].

\section{Neuroimaging acquisition and overview of multimodal image processing}

See Supplementary Material: Neuroimaging Acquisition and Structural/ Functional/Diffusion Image Processing for neuroimaging acquisition parameters and processing methods for each neuroimaging modality. A whole-brain region of interest (ROI) approach was used to create the datasets used in the DIABLO analysis. For cortical regions, the Human Connectome Project atlas [40] (HCP-MMP), which has shown to have greater amounts of reproducibility, parcellation reliability, agreement with task activation, and greatest overlap over Broadman areas compared to other major brain atlases [41], was used. For subcortical regions, the Harvard-Oxford subcortical atlas was used [42-45]. Based on these ROIs (Table 1), measures of brain morphometry, resting-state functional connectivity, and anatomical connectivity were derived. Participants' neuroimaging data were treated as separate datasets for each modality, and each dataset went through separate preprocessing methods prior to DIABLO. See "Supplementary Material: Neuroimaging data preparation for DIABLO". 
Table 1. Regions of interest.

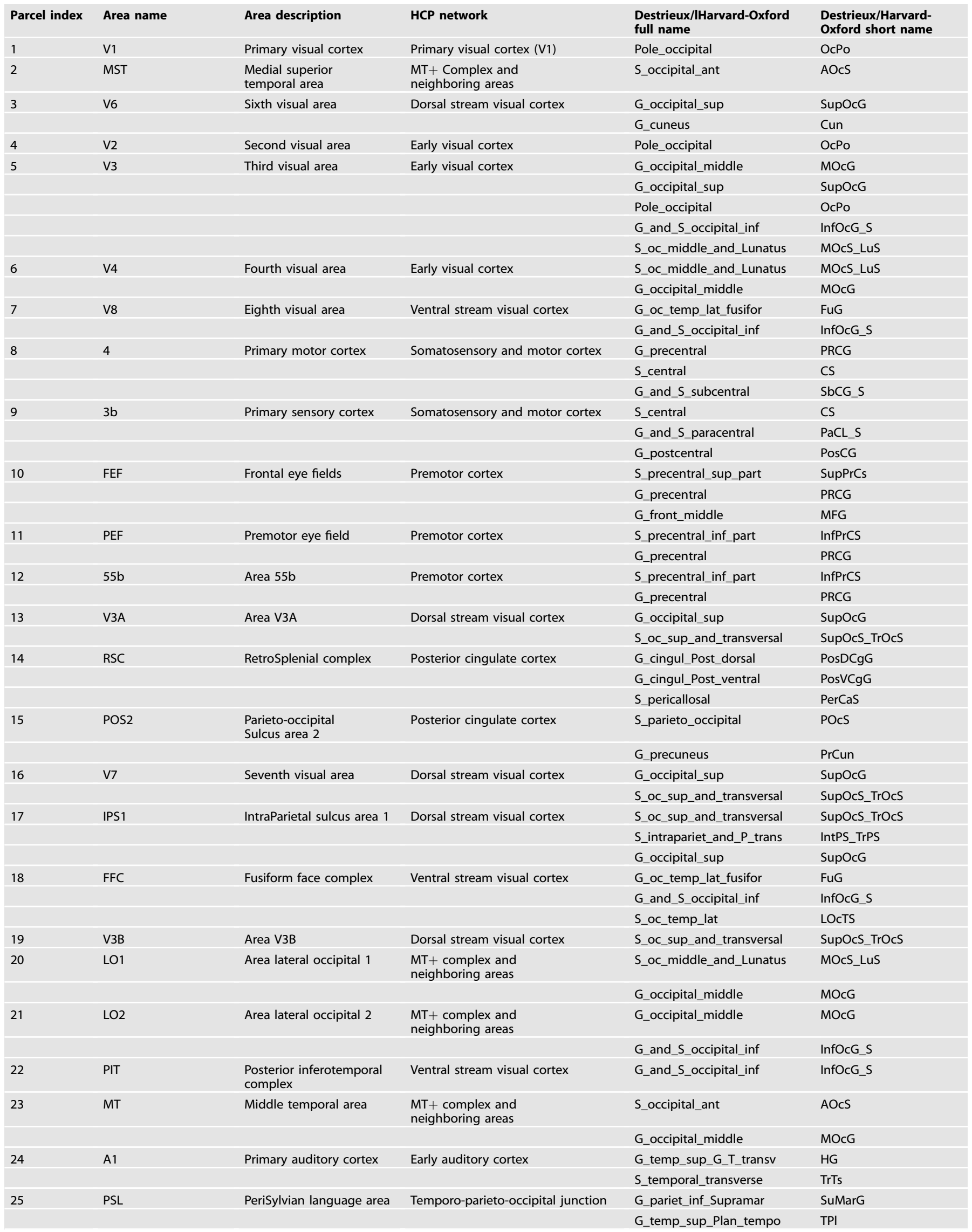


Table 1 continued

\begin{tabular}{|c|c|c|c|c|c|}
\hline Parcel index & Area name & Area description & HCP network & $\begin{array}{l}\text { Destrieux/IHarvard-Oxford } \\
\text { full name }\end{array}$ & $\begin{array}{l}\text { Destrieux/Harvard- } \\
\text { Oxford short name }\end{array}$ \\
\hline 27 & PCV & PreCuneus visual area & Posterior cingulate cortex & G_precuneus & PrCun \\
\hline \multirow[t]{2}{*}{28} & STV & $\begin{array}{l}\text { Superior temporal } \\
\text { visual area }\end{array}$ & Temporo-parieto-occipital junction & G_pariet_inf_Supramar & SuMarG \\
\hline & & & & S_temporal_sup & SupTS \\
\hline \multirow[t]{2}{*}{30} & $7 m$ & Area $7 \mathrm{~m}$ & Posterior cingulate cortex & G_precuneus & PrCun \\
\hline & & & & S_subparietal & SbPS \\
\hline \multirow[t]{2}{*}{31} & POS1 & $\begin{array}{l}\text { Parieto-occipital } \\
\text { sulcus area } 1\end{array}$ & Posterior cingulate cortex & G_precuneus & PrCun \\
\hline & & & & S_parieto_occipital & POcS \\
\hline \multirow[t]{4}{*}{33} & v23ab & Area ventral $23 \mathrm{a}+\mathrm{b}$ & Posterior cingulate cortex & G_cingul_Post_dorsal & PosDCgG \\
\hline & & & & G_cingul_Post_ventral & PosVCgG \\
\hline & & & & G_precuneus & PrCun \\
\hline & & & & S_subparietal & SbPS \\
\hline 34 & d23ab & Area dorsal $23 \mathrm{a}+\mathrm{b}$ & Posterior cingulate cortex & G_cingul_Post_dorsal & PosDCgG \\
\hline 35 & $31 \mathrm{pv}$ & Area $31 p$ ventral & Posterior cingulate cortex & S_subparietal & SbPS \\
\hline 36 & $5 \mathrm{~m}$ & Area $5 \mathrm{~m}$ & $\begin{array}{l}\text { Paracentral lobular and mid- } \\
\text { cingulate cortex }\end{array}$ & G_and_S_paracentral & PaCL_S \\
\hline 37 & $5 \mathrm{mv}$ & Area $5 \mathrm{~m}$ ventral & $\begin{array}{l}\text { Paracentral lobular and mid- } \\
\text { cingulate cortex }\end{array}$ & S_cingul_Marginalis & CgSMarp \\
\hline \multirow[t]{2}{*}{38} & $23 c$ & Area $23 c$ & Posterior cingulate cortex & G_and_S_cingul_Mid_Post & MPosCgG_S \\
\hline & & & & S_cingul_Marginalis & CgSMarp \\
\hline 40 & & & & G_and_S_paracentral & PaCL_S \\
\hline 41 & $24 d v$ & Ventral area $24 d$ & $\begin{array}{l}\text { Paracentral lobular and mid- } \\
\text { cingulate cortex }\end{array}$ & G_and_S_cingul_Mid_Post & MPosCgG_S \\
\hline 42 & $7 A L$ & Lateral area $7 \mathrm{~A}$ & Superior parietal cortex & G_parietal_sup & SupPL \\
\hline \multirow[t]{2}{*}{43} & SCEF & $\begin{array}{l}\text { Supplementary and } \\
\text { cingulate eye field }\end{array}$ & $\begin{array}{l}\text { Paracentral lobular and mid- } \\
\text { cingulate cortex }\end{array}$ & G_front_sup & SupFG \\
\hline & & & & G_and_S_cingul_Mid_Ant & MACgG_S \\
\hline \multirow[t]{2}{*}{44} & $6 m a$ & Area $6 \mathrm{~m}$ anterior & $\begin{array}{l}\text { Paracentral lobular and mid- } \\
\text { cingulate cortex }\end{array}$ & S_front_sup & SupFS \\
\hline & & & & G_front_sup & SupFG \\
\hline \multirow[t]{2}{*}{45} & $7 \mathrm{Am}$ & Medial area 7A & Superior parietal cortex & G_parietal_sup & SupPL \\
\hline & & & & G_precuneus & PrCun \\
\hline 46 & $7 P I$ & Lateral area $7 \mathrm{P}$ & Superior parietal cortex & G_parietal_sup & SupPL \\
\hline \multirow[t]{2}{*}{47} & 7PC & Area 7PC & Superior parietal cortex & G_parietal_sup & SupPL \\
\hline & & & & S_intrapariet_and_P_trans & IntPS_TrPS \\
\hline \multirow[t]{2}{*}{48} & LIPv & $\begin{array}{l}\text { Area lateral intraparietal } \\
\text { ventral }\end{array}$ & Superior parietal cortex & S_intrapariet_and_P_trans & IntPS_TrPS \\
\hline & & & & G_parietal_sup & SupPL \\
\hline \multirow[t]{2}{*}{49} & VIP & $\begin{array}{l}\text { Ventral intraparietal } \\
\text { complex }\end{array}$ & Superior parietal cortex & G_parietal_sup & SupPL \\
\hline & & & & S_intrapariet_and_P_trans & IntPS_TrPS \\
\hline \multirow[t]{2}{*}{50} & MIP & Medial intraparietal area & Superior parietal cortex & G_parietal_sup & SupPL \\
\hline & & & & S_intrapariet_and_P_trans & IntPS_TrPS \\
\hline 51 & 1 & Area 1 & Somatosensory and motor cortex & G_postcentral & PosCG \\
\hline
\end{tabular}


Table 1 continued

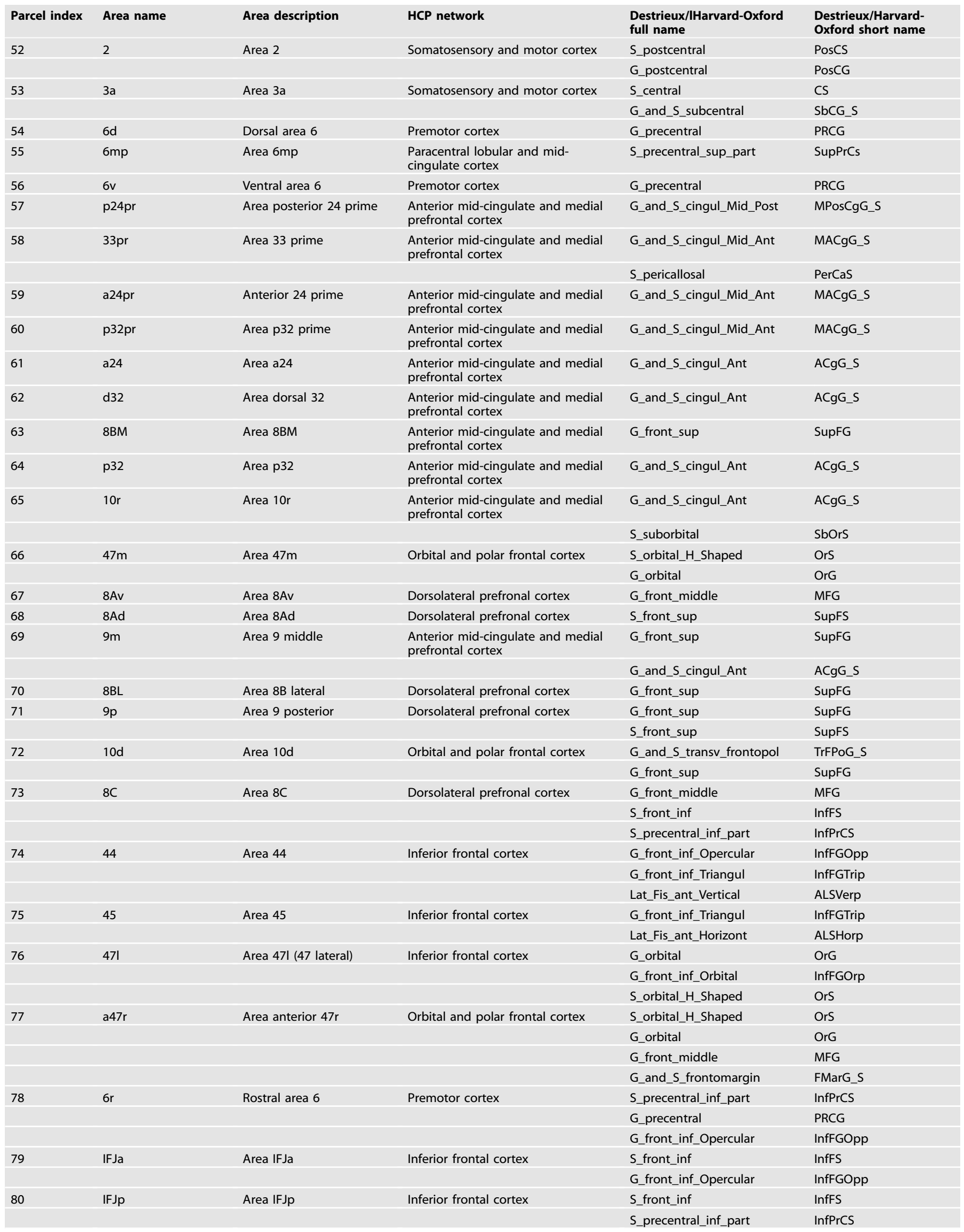


Table 1 continued

\begin{tabular}{|c|c|c|c|c|c|}
\hline Parcel index & Area name & Area description & HCP network & $\begin{array}{l}\text { Destrieux/lHarvard-Oxford } \\
\text { full name }\end{array}$ & $\begin{array}{l}\text { Destrieux/Harvard- } \\
\text { Oxford short name }\end{array}$ \\
\hline 81 & & & & G_front_inf_Triangul & InfFGTrip \\
\hline & & & & G_front_inf_Triangul & InfFGTrip \\
\hline & & & & S_orbital_lateral & LORs \\
\hline 83 & p9-46v & Area posterior $9-46 \mathrm{v}$ & Dorsolateral prefronal cortex & G_front_middle & MFG \\
\hline & & & & G_front_middle & MFG \\
\hline & & & & S_front_sup & SupFS \\
\hline & & & & S_front_middle & MFS \\
\hline \multirow[t]{2}{*}{85} & a9-46v & Area anterior $9-46 \mathrm{v}$ & Dorsolateral prefronal cortex & G_front_middle & MFG \\
\hline & & & & S_front_inf & InfFS \\
\hline 86 & $9-46 d$ & Area $9-46 d$ & Dorsolateral prefronal cortex & S_front_middle & MFS \\
\hline & & & & G_front_sup & SupFG \\
\hline & & & & S_front_sup & SupFS \\
\hline & & & & G_and_S_transv_frontopol & TrFPoG_S \\
\hline \multirow[t]{2}{*}{88} & $10 v$ & Area $10 \mathrm{v}$ & $\begin{array}{l}\text { Anterior mid-cingulate and medial } \\
\text { prefrontal cortex }\end{array}$ & G_rectus & RG \\
\hline & & & & G_and_S_frontomargin & FMarG_S \\
\hline \multirow[t]{2}{*}{89} & a10p & Area anterior $10 p$ & Orbital and polar frontal cortex & G_and_S_frontomargin & FMarG_S \\
\hline & & & & G_and_S_transv_frontopol & TrFPoG_S \\
\hline \multirow[t]{2}{*}{90} & $10 \mathrm{pp}$ & Polar $10 p$ & Orbital and polar frontal cortex & G_and_S_transv_frontopol & TrFPoG_S \\
\hline & & & & G_and_S_frontomargin & FMarG_S \\
\hline \multirow[t]{2}{*}{91} & 111 & Area 111 & Orbital and polar frontal cortex & G_orbital & OrG \\
\hline & & & & S_orbital_H_Shaped & Ors \\
\hline 95 & LIPd & $\begin{array}{l}\text { Area lateral } \\
\text { intraparietal dorsal }\end{array}$ & Superior parietal cortex & S_intrapariet_and_P_trans & IntPS_TrPS \\
\hline \multirow[t]{4}{*}{96} & $6 a$ & Area 6 anterior & Premotor cortex & S_front_sup & SupFS \\
\hline & & & & S_precentral_sup_part & SupPrCs \\
\hline & & & & G_front_middle & MFG \\
\hline & & & & G_precentral & PRCG \\
\hline \multirow[t]{2}{*}{97} & i6-8 & $\begin{array}{l}\text { Inferior 6-8 } \\
\text { transitional area }\end{array}$ & Dorsolateral prefronal cortex & G_front_middle & MFG \\
\hline & & & & S_front_sup & SupFS \\
\hline \multirow[t]{2}{*}{98} & s6-8 & $\begin{array}{l}\text { Superior } 6-8 \\
\text { transitional area }\end{array}$ & Dorsolateral prefronal cortex & G_front_sup & SupFG \\
\hline & & & & S_front_sup & SupFS \\
\hline \multirow[t]{2}{*}{99} & 43 & Area 43 & Posterior opercular cortex & G_and_S_subcentral & SbCG_S \\
\hline & & & & G_front_inf_Opercular & InfFGOpp \\
\hline 100 & OP4 & Area OP4/PV & Posterior opercular cortex & G_and_S_subcentral & SbCG_S \\
\hline & & & & G_pariet_inf_Supramar & SuMarG \\
\hline 101 & OP1 & Area OP1/SII & Posterior opercular cortex & G_pariet_inf_Supramar & SuMarG \\
\hline & & & & G_and_S_subcentral & SbCG_S \\
\hline & & & & Lat_Fis_post & PosLS \\
\hline 102 & OP2-3 & Area OP2-3/VS & Posterior opercular cortex & Lat_Fis_post & PosLS \\
\hline & & & & G_and_S_subcentral & SbCG_S \\
\hline & & & & S_circular_insula_sup & SupCirlnS \\
\hline 103 & 52 & Area 52 & Insular and frontal opercular cortex & S_circular_insula_inf & InfCirlns \\
\hline & & & & Lat_Fis_post & PosLS \\
\hline
\end{tabular}


Table 1 continued

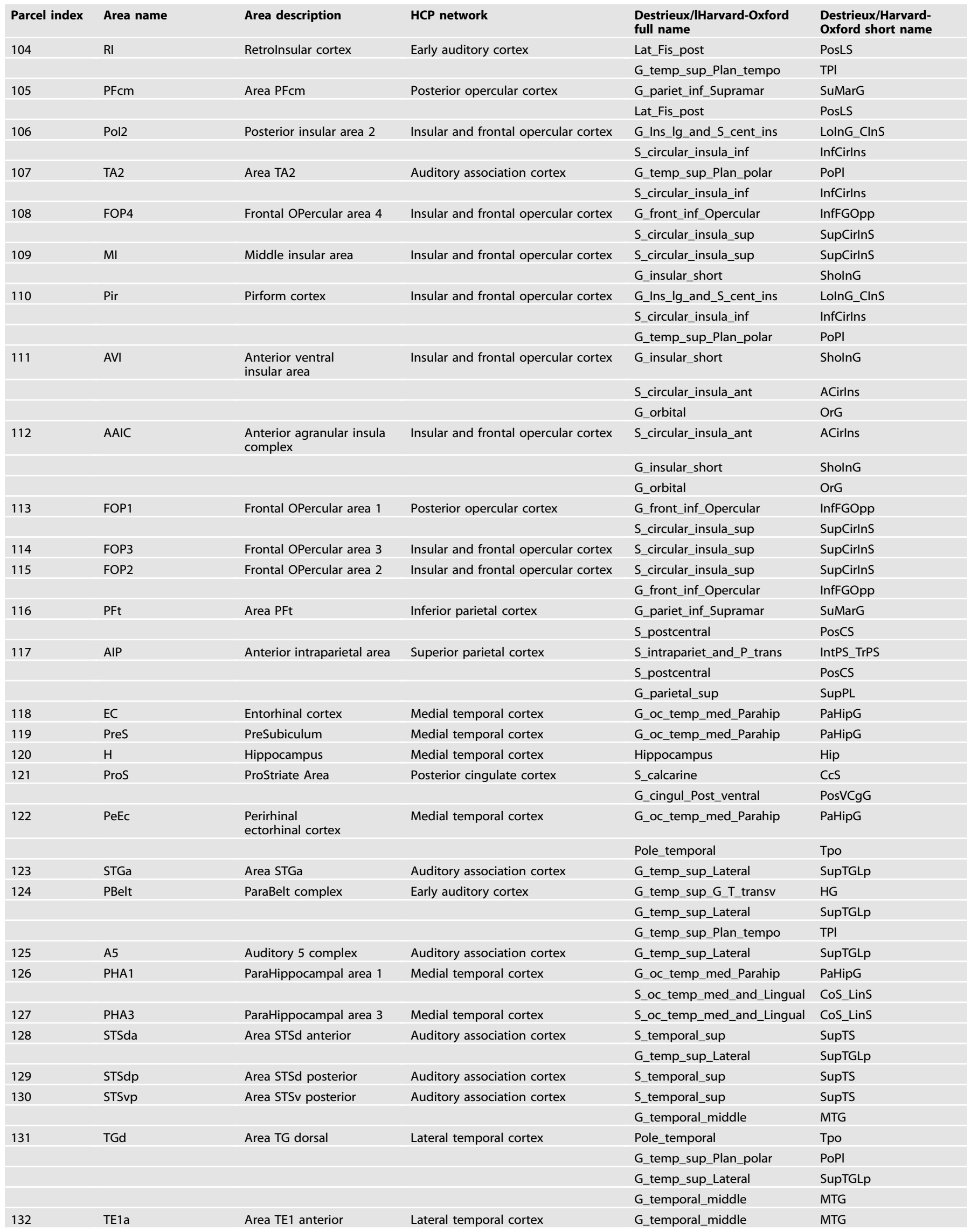


Table 1 continued

\begin{tabular}{|c|c|c|c|c|c|}
\hline Parcel index & Area name & Area description & HCP network & $\begin{array}{l}\text { Destrieux/lHarvard-Oxford } \\
\text { full name }\end{array}$ & $\begin{array}{l}\text { Destrieux/Harvard- } \\
\text { Oxford short name }\end{array}$ \\
\hline \multirow[t]{3}{*}{133} & TE1p & Area TE1 posterior & Lateral temporal cortex & G_temporal_middle & MTG \\
\hline & & & & S_temporal_inf & InfTS \\
\hline & & & & G_temporal_inf & InfTG \\
\hline \multirow[t]{3}{*}{134} & TE2a & Area TE2 anterior & Lateral temporal cortex & G_temporal_inf & InfTG \\
\hline & & & & S_temporal_inf & InfTS \\
\hline & & & & Pole_temporal & Tpo \\
\hline \multirow[t]{3}{*}{135} & TF & Area TF & Lateral temporal cortex & S_collat_transv_ant & ATrCos \\
\hline & & & & G_temporal_inf & InfTG \\
\hline & & & & G_oc_temp_lat_fusifor & FuG \\
\hline \multirow[t]{4}{*}{136} & TE2 $p$ & Area TE2 posterior & Lateral temporal cortex & S_oc_temp_lat & LOcTS \\
\hline & & & & G_temporal_inf & InfTG \\
\hline & & & & S_collat_transv_ant & ATrCos \\
\hline & & & & G_temporal_inf & InfTG \\
\hline \multirow[t]{3}{*}{137} & PHT & Area PHT & Lateral temporal cortex & G_temporal_middle & MTG \\
\hline & & & & S_temporal_inf & InfTS \\
\hline & & & & G_temporal_inf & InfTG \\
\hline \multirow[t]{3}{*}{138} & $\mathrm{PH}$ & Area $\mathrm{PH}$ & $\begin{array}{l}\text { MT+ complex and } \\
\text { neighboring areas }\end{array}$ & G_temporal_inf & InfTG \\
\hline & & & & G_and_S_occipital_inf & InfOcG_S \\
\hline & & & & S_oc_temp_lat & LOcTS \\
\hline \multirow[t]{3}{*}{139} & TPOJ1 & $\begin{array}{l}\text { Area } \\
\text { TemporoParietoOccipital } \\
\text { Junction } 1\end{array}$ & Temporo-parieto-occipital junction & S_temporal_sup & SupTS \\
\hline & & & & G_temp_sup_Lateral & SupTGLp \\
\hline & & & & G_temporal_middle & MTG \\
\hline \multirow[t]{2}{*}{140} & TPOJ2 & $\begin{array}{l}\text { Area } \\
\text { TemporoParietoOccipital } \\
\text { Junction } 2\end{array}$ & Temporo-parieto-occipital junction & S_temporal_sup & SupTS \\
\hline & & & & G_temporal_middle & MTG \\
\hline \multirow[t]{2}{*}{141} & TPOJ3 & $\begin{array}{l}\text { Area } \\
\text { TemporoParietoOccipital } \\
\text { Junction } 3\end{array}$ & Temporo-parieto-occipital junction & S_temporal_sup & SupTS \\
\hline & & & & G_occipital_middle & MOcG \\
\hline \multirow[t]{4}{*}{142} & DVT & $\begin{array}{l}\text { Dorsal transitional } \\
\text { visual area }\end{array}$ & Posterior cingulate cortex & G_parietal_sup & SupPL \\
\hline & & & & G_occipital_sup & SupOcG \\
\hline & & & & S_parieto_occipital & POcS \\
\hline & & & & S_calcarine & $\mathrm{CcS}$ \\
\hline \multirow[t]{3}{*}{143} & PGp & Area PGp & Inferior parietal cortex & S_temporal_sup & SupTS \\
\hline & & & & S_oc_middle_and_Lunatus & MOcS_LuS \\
\hline & & & & G_occipital_middle & MOcG \\
\hline \multirow[t]{3}{*}{144} & IP2 & Area IntraParietal 2 & Inferior parietal cortex & S_intrapariet_and_P_trans & IntPS_TrPS \\
\hline & & & & S_interm_prim_Jensen & JS \\
\hline & & & & G_pariet_inf_Supramar & SuMarG \\
\hline \multirow[t]{2}{*}{145} & IP1 & Area IntraParietal 1 & Inferior parietal cortex & G_pariet_inf_Angular & AngG \\
\hline & & & & S_intrapariet_and_P_trans & IntPS_TrPS \\
\hline \multirow[t]{3}{*}{146} & IPO & Area IntraParietal 0 & Inferior parietal cortex & S_intrapariet_and_P_trans & IntPS_TrPS \\
\hline & & & & S_oc_sup_and_transversal & SupOcS_TrOcS \\
\hline & & & & G_occipital_middle & MOcG \\
\hline \multirow[t]{3}{*}{147} & PFop & Area PF opercular & Inferior parietal cortex & G_pariet_inf_Supramar & SuMarG \\
\hline & & & & G_and_S_subcentral & SbCG_S \\
\hline & & & & G_postcentral & PosCG \\
\hline \multirow[t]{3}{*}{148} & PF & Area PF complex & Inferior parietal cortex & G_pariet_inf_Supramar & SuMarG \\
\hline & & & & Lat_Fis_post & PosLS \\
\hline & & & & G_temp_sup_Plan_tempo & TPI \\
\hline \multirow[t]{4}{*}{149} & PFm & Area PFm complex & Inferior parietal cortex & G_pariet_inf_Supramar & SuMarG \\
\hline & & & & S_interm_prim_Jensen & JS \\
\hline & & & & S_temporal_sup & SupTS \\
\hline & & & & G_pariet_inf_Angular & AngG \\
\hline \multirow[t]{2}{*}{150} & PGi & Area PGi & Inferior parietal cortex & S_temporal_sup & SupTS \\
\hline & & & & G_pariet_inf_Angular & AngG \\
\hline
\end{tabular}


Table 1 continued

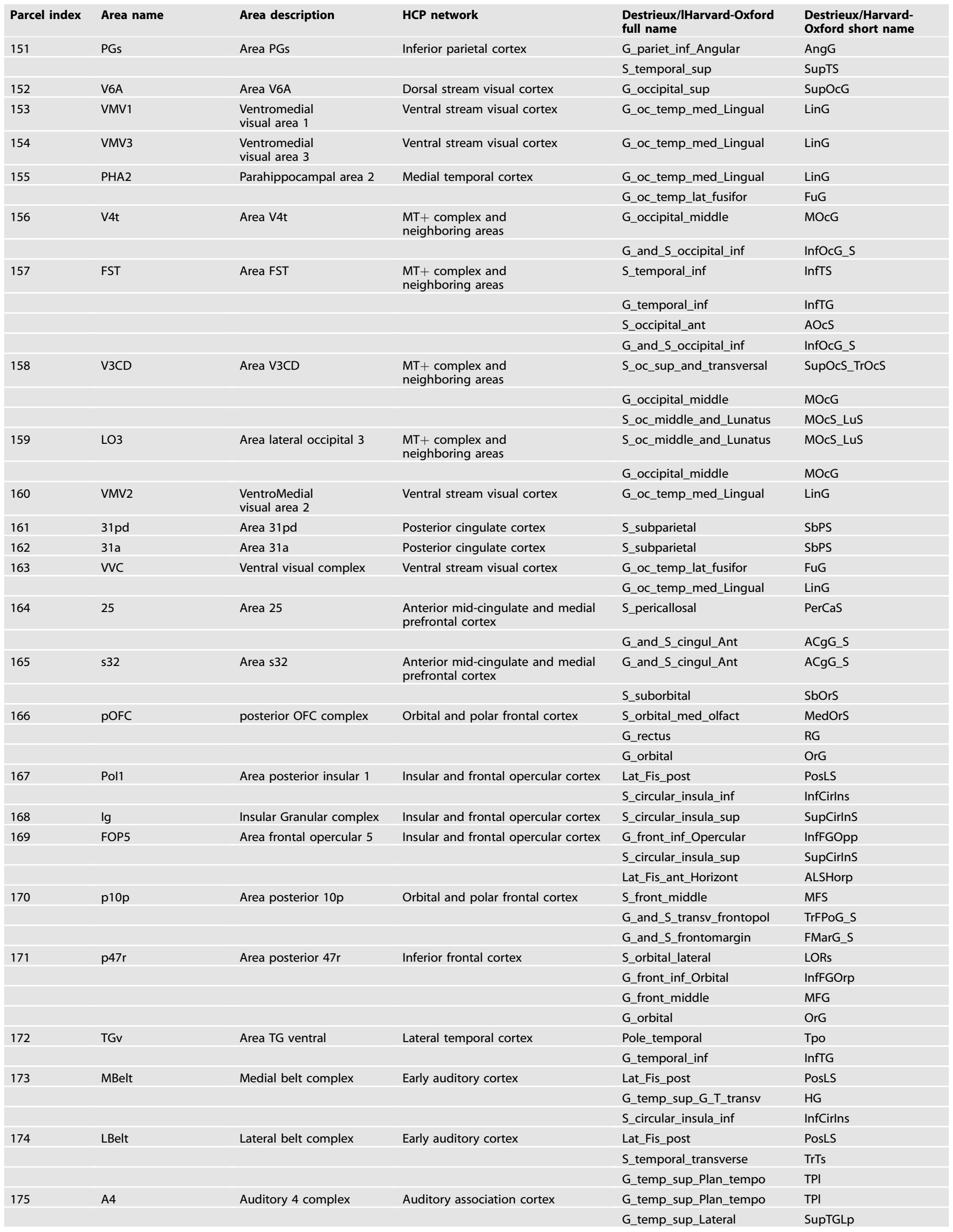


Table 1 continued

\begin{tabular}{|c|c|c|c|c|c|}
\hline Parcel index & Area name & Area description & HCP network & $\begin{array}{l}\text { Destrieux/lHarvard-Oxford } \\
\text { full name }\end{array}$ & $\begin{array}{l}\text { Destrieux/Harvard- } \\
\text { Oxford short name }\end{array}$ \\
\hline 176 & STSva & Area STSv anterior & Auditory association cortex & S_temporal_sup & SupTS \\
\hline \multirow[t]{2}{*}{177} & TE1m & Area TE1 middle & Lateral temporal cortex & G_temporal_middle & MTG \\
\hline & & & & S_temporal_inf & InfTS \\
\hline 178 & $\mathrm{PI}$ & Para-insular area & Insular and frontal opercular cortex & S_circular_insula_inf & InfCirlns \\
\hline \multirow[t]{2}{*}{179} & a32pr & Area anterior 32 prime & $\begin{array}{l}\text { Anterior mid-cingulate and medial } \\
\text { prefrontal cortex }\end{array}$ & G_and_S_cingul_Ant & ACgG_S \\
\hline & & & & G_and_S_cingul_Mid_Ant & MACgG_S \\
\hline \multirow[t]{3}{*}{180} & p24 & Area posterior 24 & $\begin{array}{l}\text { Anterior mid-cingulate and medial } \\
\text { prefrontal cortex }\end{array}$ & S_pericallosal & PerCaS \\
\hline & & & & G_and_S_cingul_Ant & ACgG_S \\
\hline & & & & G_and_S_cingul_Mid_Ant & MACgG_S \\
\hline \multirow[t]{14}{*}{ N/A } & $N / A$ & $\mathrm{~N} / \mathrm{A}$ & $\mathrm{N} / \mathrm{A}$ & Cerebellar cortex & Cerebellum_Cortex \\
\hline & & & & Thalamus proper & Thalamus_Proper \\
\hline & & & & Caudate nucleus & Caudate \\
\hline & & & & Putamen & Putamen \\
\hline & & & & Pallidum & Pallidum \\
\hline & & & & Amygdala & Amygdala \\
\hline & & & & (Nucleus) Accumbens area & Accumbens_area \\
\hline & & & & Cerebellar cortex & Cerebellum_Cortex \\
\hline & & & & Thalamus proper & Thalamus_Proper \\
\hline & & & & Caudate nucleus & Caudate \\
\hline & & & & Putamen & Putamen \\
\hline & & & & Pallidum & Pallidum \\
\hline & & & & Amygdala & Amygdala \\
\hline & & & & (Nucleus) Accumbens area & Accumbens_area \\
\hline AAN Name & AAN description & AAN hemisphere name & & & AAN hemisphere \\
\hline DR & Dorsal raphé nuclei & DR & & & B \\
\hline LC & Locus coeruleus & L_LC & & & $\mathrm{L}$ \\
\hline LC & Locus coeruleus & R_LC & & & $\mathrm{R}$ \\
\hline MRF & $\begin{array}{l}\text { Mesencephalic } \\
\text { reticular formation }\end{array}$ & L_MRF & & & $\mathrm{L}$ \\
\hline MRF & $\begin{array}{l}\text { Mesencephalic } \\
\text { reticular formation }\end{array}$ & R_MRF & & & $\mathrm{R}$ \\
\hline MR & Median raphé nuclei & MR & & & B \\
\hline PAG & Periaqueductal gray & PAG & & & B \\
\hline PBC & Parabrachial complex & L_PBC & & & $\mathrm{L}$ \\
\hline PBC & Parabrachial complex & R_PBC & & & $\mathrm{R}$ \\
\hline $\mathrm{PO}$ & Pontis oralis & L_PO & & & $\mathrm{L}$ \\
\hline $\mathrm{PO}$ & Pontis oralis & R_PO & & & $\mathrm{R}$ \\
\hline PPN & $\begin{array}{l}\text { Pendunculopontine } \\
\text { nucleus }\end{array}$ & L_PPN & & & $\mathrm{L}$ \\
\hline PPN & $\begin{array}{l}\text { Pendunculopontine } \\
\text { nucleus }\end{array}$ & R_PPN & & & $\mathrm{R}$ \\
\hline VTA & $\begin{array}{l}\text { Ventral } \\
\text { tegmental area }\end{array}$ & VTA & & & B \\
\hline
\end{tabular}

\section{DIABLO analysis}

Data were split into training and testing datasets ("Supplementary Information: Training and Testing Sets"). A data integration analysis for biomarker discovery using latent components (DIABLO) was conducted to achieve our study aims of predicting groups of improvers vs. nonimprovers at 3 and 12 months. The method identifies a limited number of correlated variables from multiple datasets to predict outcome. In this study, the outcome was "symptom status". The method is an extension of sparse generalized canonical correlation analysis [46], which is a generalization of partial least square for multiple matching data sets $(Q)$, to a supervised learning framework [26, 47]. Before proceeding with DIABLO analysis, individual sPLS models were run between pairwise datasets (e.g., morphometric and clinical; morphometric and resting state) to understand major sources of variation in each dataset, and guide the integration process by obtaining correlations to employ in a data-driven weighted design matrix [26]. The design matrix is a $Q \times Q$ matrix representing if and by how much each dataset should be correlated for the model's algorithms in the DIABLO analysis. Values range from 0 to 1 . The design matrix was created by taking the correlated values of the first component from each individual SPLS model (Supplementary Information, Figs. 2-13). All correlations were above 0.8 ; therefore, values were set to 1 in the design matrix (Supplementary Information: Supplemental Fig. 1) [26]. Once the design matrix was determined, A DIABLO model with five components was first fit without any variable selection, and global performance was assessed using leave-one-out-cross validation (LOOCV). The number of components chosen based on the lowest balanced error rate (BER) and distance metric (maximum distance vs centroids distance vs. mahalanobis distance) across a number of components. After determining the number of components to use, the optimal number of variables to be 
kept per component, one component at a time, by defining a grid of values -in this case from 2 to 300 - for each component. LOOCV was run with the distance metric defined above to give the lowest BER. The classification error rate was then extracted averaged across every LOOCV model for each tested grid value. The optimal number of components, and features per component was then extracted. Since this process may lead to overfitting, manual tuning of the number of features per component was then conducted to get the lowest balanced error rate and best accuracy on a holdout testing dataset. The main output measures for DIABLO are a set of components (i.e., latent variables) chosen in the model, a set of loading vectors (i.e., coefficients assigned to each variable to define each component), and a list of selected variables from each dataset and associated to each component. Loadings are the coefficients assigned to each variable to define each component, and their absolute value represents the importance of each variable in DIABLO. It is important to note that each loading vector is assigned to a particular component, and the loading vectors are obtained so that the covariance between a linear combination of $X$ variables and $Y$ is maximized. Individual sample plots represent each individual projected onto a space that is defined by the components. The coordinates for each individual are determined by their component values/scores. Loading plots help visualize each coefficient (i.e., importance) assigned to the variables in each component of each dataset. Circos diagrams are built on a similarity matrix [48] and represent the correlation between variables from different datasets, and a cutoff was chosen as $r=0.7$ as this is universally considered a "strong" correlation. Relevance networks are graphs where the nodes represent the variables chosen by DIABLO and the edges represent variable associations. Edges between nodes were only drawn if the association was 0.7 or higher. Nodes/variables extracted from DIABLO that did not have any associations were not represented on the network. The area under the receiver operating characteristic (ROC) curve was calculated for each dataset separately by component, and $p$-values were calculated using the Wilcoxon test comparing improvers vs. non-improvers. The area under the ROC curve is a way to summarize the overall diagnostic accuracy of the model. The values range from 0 to 1 , where 1 represents a perfectly accurate model. A value of 0.5 represents no discriminatory ability. Values between 0.7 and 0.8 are considered acceptable, while between 0.8 and 0.9 is considered excellent, and greater values are considered outstanding [49]. The model was then tested on the testing data and peformance assessed with the BER, along with confusion matrix statistics such as sensitivity, specificity and the F1 statistic.

\section{RESULTS}

See Supplementary Information, Tables 2 and 3 for descriptive statsitics. An independent $t$-test was done to confirm that age was similar in the train $(M=28.96, S D=10.97)$ and test $(M=29.64, S D$ $=14.04)$ datasets $(t=-0.15, p=0.88, d=0.06)$.

\section{Symptom change validation across 12-months}

To understand the rate of improvement, 24 participants $(40 \%)$ showed improvement after 3 months and 17 participants $(40 \%)$ improved after 12 months. To validate that the 12-month improvers were improving over 12 months compared to nonimprovers, the percentage of timepoints $(3,6,9$, and 12 months) where subjects "improved" (e.g., decreased on the IBS-SSS by 50 points) from baseline was first calculated. Over 12 months, compared to non-improvers $(M=22 \%, S D=23 \%)$, improvers $(M$ $=71 \%, \mathrm{SD}=22 \%$ ) had a much higher percentage of time points showing improvement $\left(t_{(41)}=6.92, p<0.001, d=2.18\right)$. Of the 17 patients that improved after 12 months, 11 of them improved after 3 months. In the 26 patients that did not improve after 12 months, eight patients improved after 3 months.

\section{DIABLO results}

DIABLO successfully identified a correlated 'omics signature from baseline using multimodal imaging and clinical and behavioral assessments by classifying groups of improvers and non-improvers at 3 and 12 months. Based on the balanced error rate (i.e., the average proportion of wrong classifications in each class validated via LOOCV in the training sample), three and two components were selected for the 3 and 12 month models, respectively. Following feature tuning and selection, and validation of the final model with LOOCV, both models achieved an AUROC over 0.88 $(p<0.05)$. (Supplementary Information, Tables 3 and 4).

For the DIABLO model predicting three-month symptom changes, the tuning process on the training set identified a multiomics signature of three components. Component one had five morphometry, four anatomical connectivity, four resting-state functional connectivity, and two clinical features. Component two had four morphometry, three anatomical connectivity, three resting-state functional connectivity, and three clinical features. Component three had two morphometry, two anatomical connectivity, three restingstate functional connectivity, and three clinical features.

For the DIABLO model predicting 12-month symptom changes, the signature was composed of two components. Component 1 had four morphometry, five anatomical connectivity, three resting-state functional connectivity, and three clinical features. Component 2 had two morphometry, three anatomical connectivity, two resting-state functional connectivity, and four clinical features.

Areas under the ROC curve (AUROC) by data type, and of the final DIABLO model show high classification accuracy (Supplementary Information, Tables 3 and 4). Predictions and confusion matrix statistics were then assessed using the final model on an external test dataset (Fig. 1).

\section{Prediction of 3-month improver model based on external test set and contributing features}

The 3-month DIABLO model on training data was used to predict if groups of patients can be classified as improvers or non-improvers on an independent testing dataset consisting of multimodal brain and clinical data. The model predicted classes on the test dataset with $91 \%$ accuracy and $F 1=0.85$ (Fig. 1A). Features that contribute to the model, and the group expressing the maximal value on components $1-3$ can be seen in Fig. 2A-C. Sample plots in the three component space for each data type can be seen in Fig. 3A. The circos diagram in Fig. 3B shows all of the features of importance selected by DIABLO, which features are greatly correlated, and the relative mean values of each feature in each group. The relevance network in Fig. $3 C$ shows only the variables that are highly correlated with each other for easier viewing, along with box-violin plots showing the distribution of each feature per group. "Supplementary Information: Distribution of Selected Markers by DIABLO-3 months".

On component 1, the morphometry features consisted of surface area and volume of the superior parietal cortex and the posterior cingulate (PCC), both hubs of the default mode network. The anatomical connectivity features consisted of connectivity within the PCC, between the PCC and paracentral lobular cortex, between the bilateral aMCC, and between the hippocampus and visual cortex. The rs-FC features consisted rs-FC within and between the lateral temporal cortices of the default mode network and between the PCC and fusiform face gyrus. Clinical features consisted of the general subscale and total score of the early life trauma questionnaire.

On component 2, the morphometry features consisted of volume of the anterior insula (aINS), mean curvature of the orbitofrontal cortex (OFC), volume in the lateral occipital cortex and surface area in the premotor cortex. Anatomical connectivity features consisted of connectivity within the dorsolateral prefrontal cortex, between the thalamus and caudate nucleus, and between the third visual area and auditory association cortex. The rs-FC features consisted of rs-FC within the visual cortex, between the inferior parietal cortex and secondary somatosensory cortex, and between the subcentral gyrus and operculum. Clinical features included the CD-RISC Persistence and total scales, and lower scores on the ETI physical subscale. 


\section{Confusion Matricies - Testing Data}
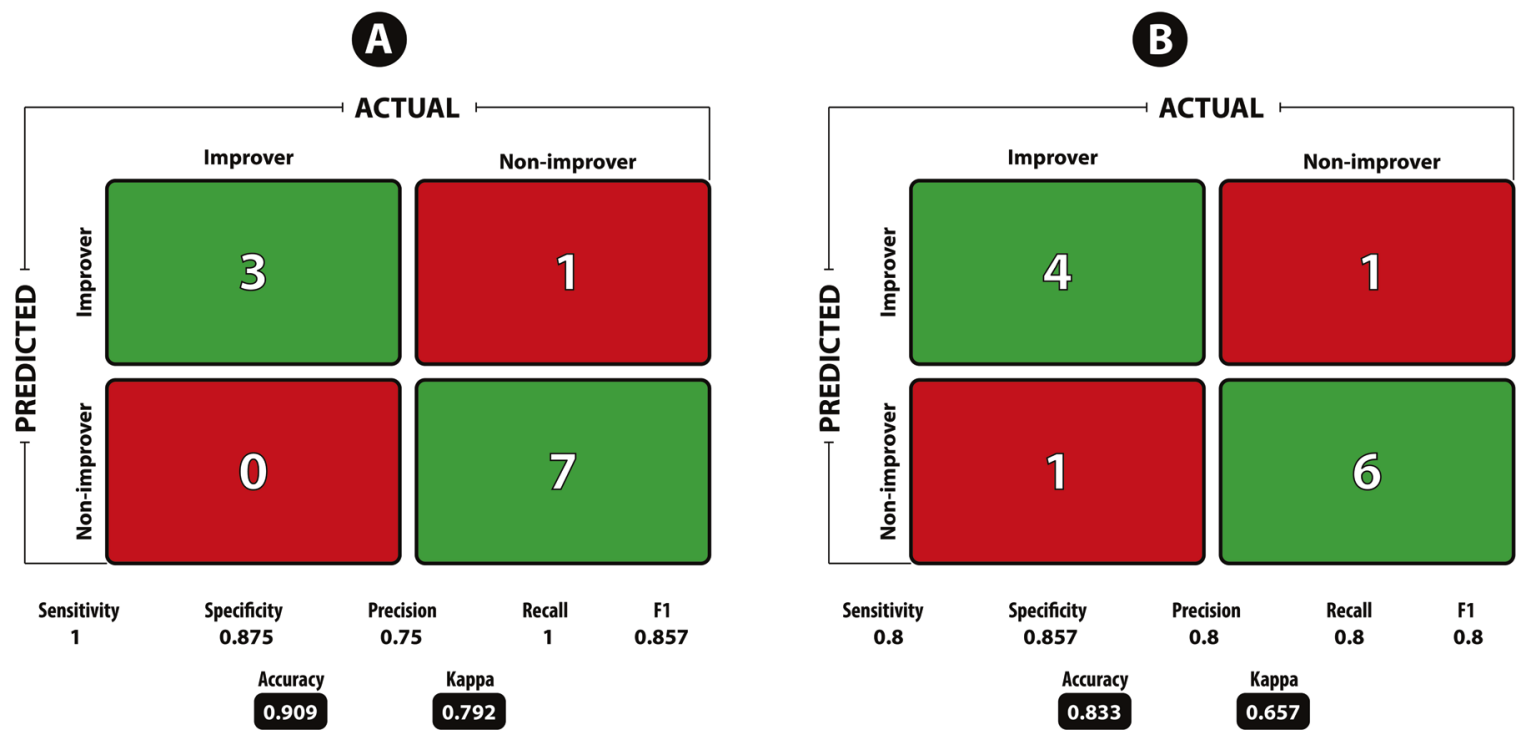

Fig. 1 Confusion matrix statistics on the testing dataset for the model predicting 3-month and 12-month improvers and non-improvers. Sensitivity and recall are defined as the true positive rate (i.e., number of predicted improvers divided by the total number of improvers), Specificity is defined as the true negative rate (i.e., number of predicted non-improvers divided by the total number of non-improvers). Precision is the ability of the classifier to not label a true negative as a positive (i.e., the ability to not label a non-improver an improver). The $F 1$ score is the harmonic mean of precision and recall, with values closer to 1 being a better score. Accuracy is defined as the number of true positives and true negatives divided by the total population. The Kappa statistic is known to be a better measure compared to accuracy, especially in the case of imbalanced classes. Kappa values between 0.61 and 0.80 are said to be "Substantial" and between 0.81 and 1.0 to be "Almost Perfect".

A
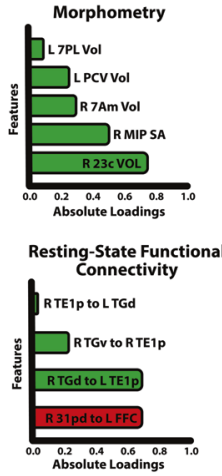
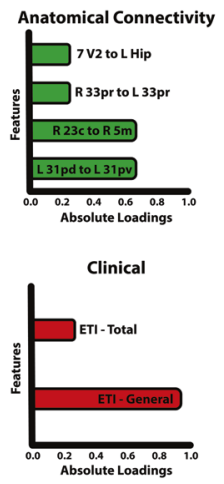

B
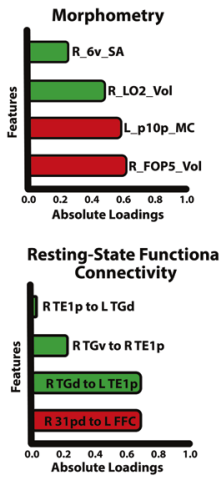

Non-Improvers
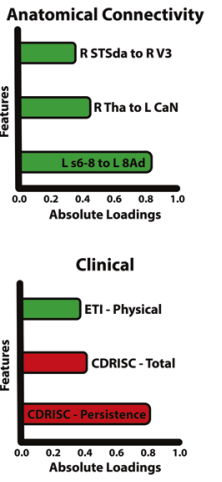

C
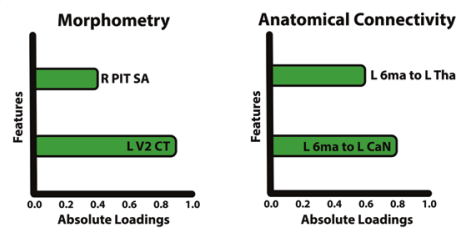

Resting-State Functional
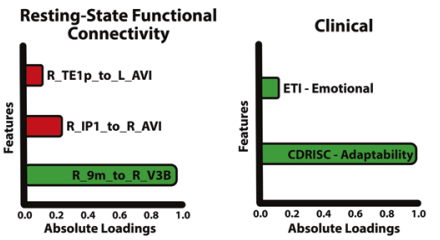

Improvers

Fig. 2 Features contributing to the multi-modal neuropsychosocial signature predicting improvers vs. non-improvers in IBS symptoms after 3 months. Absolute loadings depict the relative importance of each feature. Colors represent which group has the higher mean value for that feature. A Component 1 Morphometry: 23c Area 23C (posterior cingulate cortex [PCC]), MIP medial intraparietal area; 7 Am medial area 7A (SPC); PCV precuneus, 7 PL lateral area 7P (superior parietal cortex [SPC]). Anatomical Connectivity: 31pd area 31pd (PCC), $31 \mathrm{pv}$ area $31 \mathrm{p}$ ventral (PCC), 23c area $23 \mathrm{c}(\mathrm{PCC}), 5 \mathrm{~m}$ area $5 \mathrm{~m}$ (paracentral lobule); $33 \mathrm{pr}$ area 33 prime, V2 second visual area, Hip Hippocampus. Resting-State Functional Connectivity: 31 pd area 31 pd (PCC), FFC fusiform face cortex, TGd area TG dorsal (LTC), TE1p area TE1 posterior (LTC), TGv area TG ventral. Clinical: ETI general early trauma inventory general score, ETI total early trauma inventory total score B: Component 2 Abbreviations: Clinical: CDRISC persistence Connor-Davidson resilience persistence subscale, CDRISC total ConnorDavidson resilience persistence total score, ETI physical early trauma inventory physical score. Morphometry: FOP5 area frontal opercular 5 , p10p area posterior 10p, LO2 area lateral occipital 2; $6 \mathrm{v}$ ventral area 6 . Anatomical connectivity: s6-8 superior 6-8 transitional area, 8Ad area 8Ad, Tha. Thalamus, CaN Caudate nucleus, STSda area STSd anterior, V3 third visual area. Resting-State Functional Connectivity: IPS1 intraparietal sulcus area 1, V4 fourth visual area, PFt area PFt, area OP1 area OP1/SII, PFop area PF opercular, OP4 area OP4/PV C: Component 3 Abbreviations: Clinical: CDRISC adaptability Connor-Davidson resilience adaptability subscale, ETI emotional early trauma inventory emotional score. Morphometry: V2 second visual area, PIT posterior inferotemporal cortex. Anatomical Connectivity: 6 ma area $6 \mathrm{~m}$ anterior, CaN Caudate nucleus, Tha Thalamus. Resting-State Functional Connectivity: $9 \mathrm{~m}$ area 9 middle, IP1 area intraparietal 1, AVI anterior ventral insular area, TE1p area TE1 posterior. 

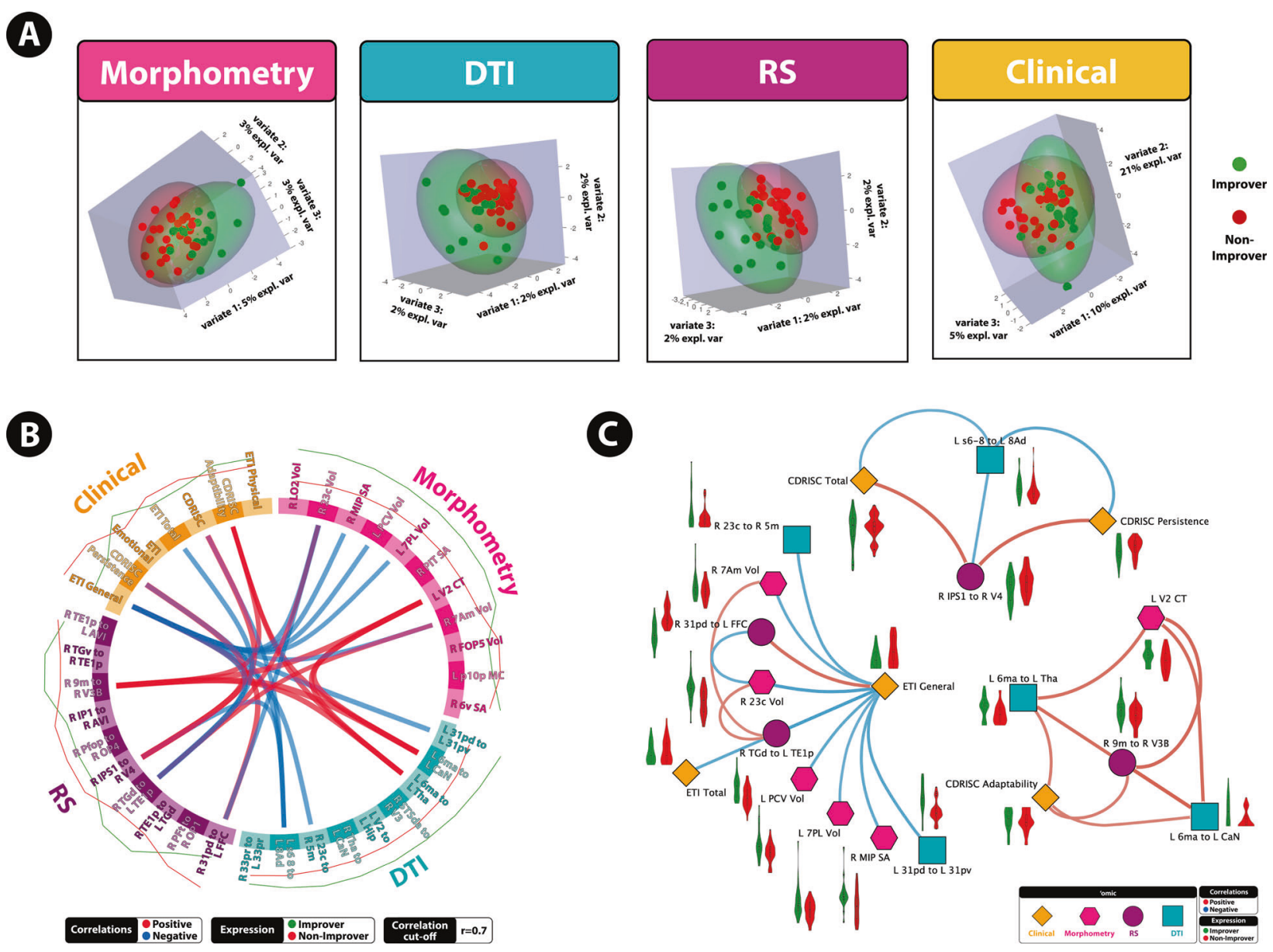

Fig. 3 A highly correlated multi-modal, brain-clinical signature predicts 3 month IBS symptom trajectories via DIABLO. A Sample plots for morphometry, anatomical connectivity, resting-state functional connectivity, and clinical variables for the 3 month DIABLO analysis. Samples are represented as points according to their projection across three latent variables. B Circos plot for the 3 month DIABLO analysis representing all of the features in the DIABLO model, and the correlations between variables of different data types. Correlation cut-off is set to $r=0.7$. Lines along the outside of the circle represent the mean "expression" levels. Greater levels are in accordance to the line being farther away from the circle. C Relevance network for the 3 month DIABLO analysis representing the correlation between variables of different data types. Red lines represent positive correlations and blue lines represent negative correlations. Boxplots with violin plots represent the distribution of each group for each feature. Correlation cut-off is set to $r=0.7$.

On component 3, the morphometry features consisted of volumes in the second visual area and posterior inferotemporal complex. Anatomical connectivity features consisted of connectivity between the supplementary motor area (SMA) and caudate nucleus, and between the SMA and thalamus. The rs-FC features included rs-FC between the medial prefrontal cortex (mPFC) and visual cortex and between the anterior insula and lateral temporal cortices. Clinical features included the CMSI-adaptability scale and ETI-emotional scale.

\section{Prediction of 12-month improver model based on external test set and contributing features}

The tuned 12-month DIABLO model on the training data was used to predict if patients can be classified as improvers or non-improvers on an independent test dataset consisting of multimodal brain and clinical data. The model predicted classes on the test dataset with $83 \%$ accuracy and $F 1=0.80$ (Fig. 1 B). Features that contribute to the model, and the group expressing the maximal value on components 1-2 can be seen in Fig. 4A, B. Sample plots in the 2 component space for each data type can be seen in Fig. 5A. The circos diagram in Fig. $5 B$ shows all of the features of importance selected by DIABLO, which features are greatly correlated, and the relative mean values of each feature in each group. The relevance network in Fig. 5C shows only the variables that are highly correlated with each other for easier viewing, along with box-violin plots showing the distribution of each feature per group. All distributions are also available in the "Supplementary Information: Distribution of Selected Markers by DIABLO-12 Months".

On component 1, morphometry features consisted of surface area and volume in the dIPFC and medial prefrontal cortex (mPFC). Anatomical connectivity features consisted of connectivity between the left and right $\mathrm{mPFC}$, and between the OFC and MPFC, between the angular gyrus and lateral temporal cortex, the anterior insula and lateral temporal cortex, within the visual cortex, between the mPFC and OFC, and within the MPFC. The rs-FC features consisted rs-FC between the SMA and premotor cortex, between the lateral temporal cortex and DIPFC, and inferior frontal cortex and subgenual ACC. Clinical features consisted of the ETI Emotional subscale, ETI total score, and the CMSI-12 months scale.

On component 2, morphometry features included volume of the superior temporal gyrus, and volume of the anterior insula. Anatomical connectivity features consisted of connectivity between the inferior parietal cortex and subcentral area, between the premotor cortex and central sulcus, and within the posterior cingulate. The rs-FC features included rs-FC between the superior parietal cortex and thalamus, and between the alNS and premotor cortex. Clinical features consisted of pain threshold, tolerance and unpleasantness ratings, BSQ overall symptoms and abdominal pain. 
(A)

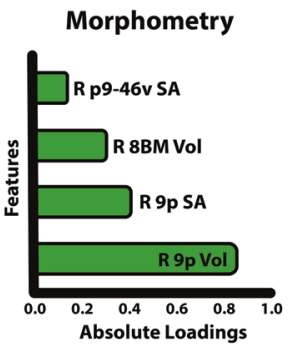

Anatomical Connectivity
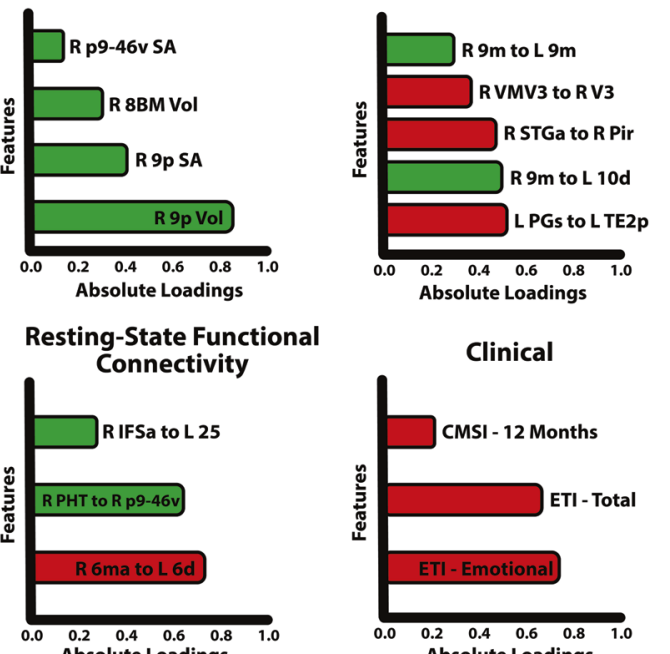

Absolute Loadings

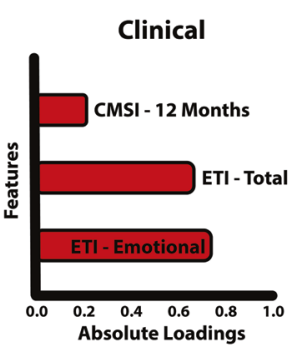

Non-Improvers
B
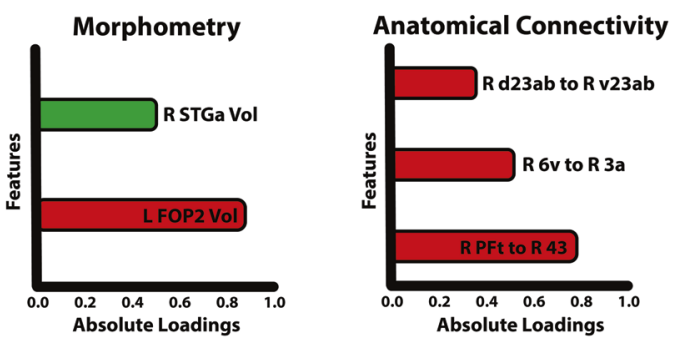

Resting-State Functional
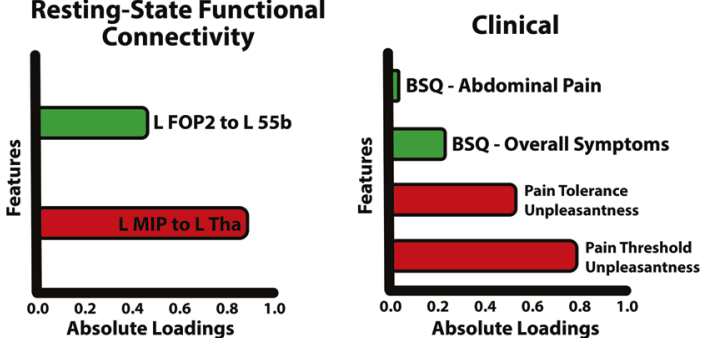

Improvers

Fig. 4 Features contributing to the multi-modal neuropsychosocial signature predicting improvers vs. non-improvers in IBS symptoms after 12 months. Absolute loadings depict the relative importance of each feature. Colors represent which group has the maximal mean value for that feature. Component 1 Abbreviations: Morphometry: 9p area 9 posterior (dIPFC), 8BM area 8BM (mPFC), p9-46v area posterior 9-46v (dIPFC). Anatomical Connectivity: $9 \mathrm{~m}$ area 9 middle (medial prefrontal cortex [mPFC]), VMV3 ventromedial visual area 1, V3 third visual area, STGa area STGa, Pir Pirform cortex (anterior insula [aINS]), 10d area 10d (OFC), PGs area PGs; TE2p area TE2 posterior Resting-State Functional Connectivity: $6 \mathrm{ma}$ area $6 \mathrm{~m}$ anterior, 6d dorsal area 6, PHT area PHT, p9-46v area posterior 9-46v, IFSa area IFSa, 25 Area 25 Clinical: ETI total early trauma inventory total score, ETI emotional early trauma inventory emotional subscale score, CMSI 12 months complex multi-symptom inventory in the past 12 months. Component 2: Abbreviations: Morphometry: FOP2 frontal opercular area 2; STGa area STGa Anatomical Connectivity: PFt area Pft; 43 area 43; 6v ventral area 6, d23ab area dorsal 23 a + b, v23ab area ventral dorsal a + b Resting-State Functional Connectivity: MIP medial intraparietal area, Tha Thalamus, FOP2 frontal opercular area 2,55b area 55b Clinical: pain threshold unpleasantness, pain tolerance unpleasantness; BSQ overall symptoms Bowel Symptom Questionnaire (overall symptom score), BSQ abdominal pain Bowel Symptom Questionnaire (overall abdominal pain).

\section{DISCUSSION}

A neuropsychosocial signature predicts improvement in patients with IBS after 3 and 12 months

With past research on pain perception and attentional systems $[10,50-52]$, these results suggest attentional systems are not able to focus away due to a greater signaling and salience of visceral nociception and perception, and lower capability of the default mode network to help take attention away from the present sensory world $[52,53]$. Therapies that target these systems, such as cognitivebehavioral therapy and practicing mindfulness [54] may be good candidates for more comprehensive IBS treatments. Specific findings within the DIABLO signature support this hypothesis.

Results showed IBS participants that did not improve in symptom severity over 12 months had lower morphological integrity, anatomical connectivity and resting-state functional connectivity within the default mode network, and reported more early life trauma. They also had lower connectivity in the dIPFC, which is responsible for inhibitory control systems, and were associated with greater persistence scores. These may be indicative of more stressful and traumatic life histories. Sensorimotor and salience systems were more greatly connected and active in patients who did not improve, and positively associated with ratings of experimental pain threshold unpleasantness. The combination of these findings indicates that attentional systems may be compromised to disengage from visceral sensations. Thus, an increase in salience and sensorimotor processing with an increased affective response to pain, and early life trauma may contribute to persistence of pain. DIABLO indicated that a multivariate signature composed of latent variables of structural, anatomical connectivity, restingstate functional connectivity and clinical/behavioral data can predict longitudinal symptom improvement in patients with IBS with $88-91 \%$ accuracy.

IBS participants whose symptoms improved-based on a 50 point decrease on the IBS-SSS from baseline after both follow-ups -had distinct neuropsychosocial patterns distinguishing them from participants with persistent or worsening symptoms. The pertinent multimodal features in each DIABLO model, from all modalities, involved key regions of the default mode network (DMN) including the posterior cingulate cortex (PCC) and dorsomedial prefrontal cortex (dmPFC), each are involved in pain perception $[50,53]$. These key hubs are part of the "dynamic pain connectome", and are activated when attention is engaged with thoughts away from present sensory stimuli and engaged in mind wandering (i.e., thoughts unrelated to the present sensory environment) $[52,53,55]$. Results indicated that regardless of short-term or long-term improvement, greater early-life trauma was associated with lower morphological integrity, anatomical connectivity, and resting-state functional connectivity within the DMN. Conversely, these hubs are deactivated when attention is placed on painful sensations [55]. The lower amount of structural integrity, anatomical connectivity, and resting-state functional connectivity within the DMN may represent deficits in disengagement from unpleasant visceral sensations. These deficits are associated with greater amounts of early life adversity (ELA), which is a risk factor for IBS $[13,56-58]$. Children and adolescents with IBS have reduced gray matter and greater resting-state connectivity across networks associated with altered pain sensitivity $[59,60]$. This study's results are consistent with previous research, demonstrating that attentional systems may be less prone to mind-wandering, which has been shown to be a key mechanism of pain inhibition $[50,55]$. Past research investigating the default 

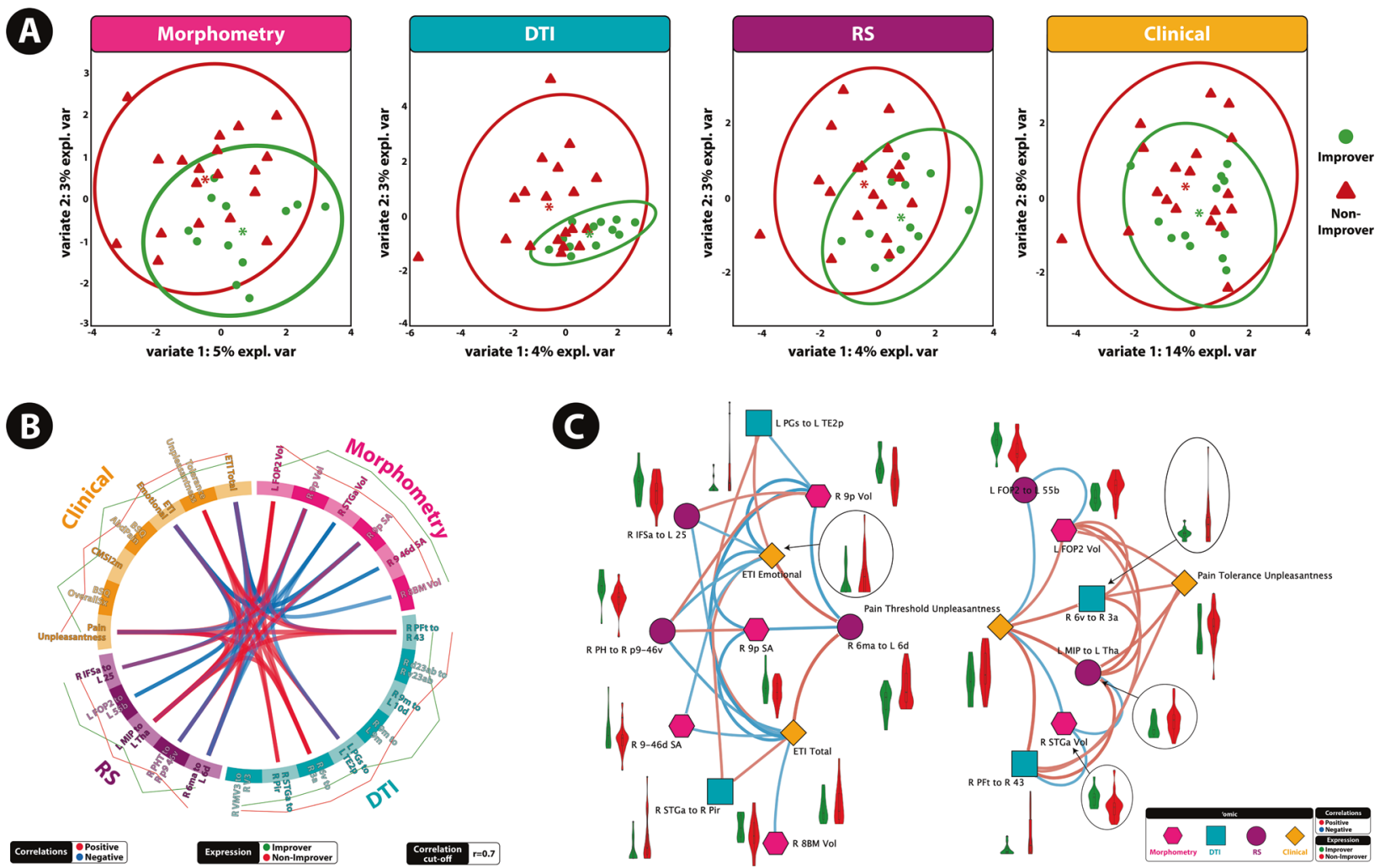

Fig. 5 A highly correlated multi-modal, brain-clinical signature predicts 12 month IBS symptom trajectories via DIABLO. A Sample plots for morphometry, anatomical connectivity, resting-state functional connectivity, and clinical variables for the 12-month DIABLO analysis. Samples are represented as points according to their projection across two latent variables. Explained variance across each component is listed. B Circos plot for the 12-month DIABLO analysis representing all of the features in the DIABLO model, and the correlations between variables of different data types. Correlation cut-off is set to $r=0.7$. Lines along the outside of the circle represent the mean "expression" levels. Greater levels are in accordance to the line being farther away from the circle. C Relevance network for the 12-month DIABLO analysis representing the correlation between variables of different data types. Red lines represent positive correlations and blue lines represent negative correlations. Boxplots with violin plots represent the distribution of each group for each feature. Correlation cut-off is set to $r=0.7$.

mode network in patients with IBS, has shown rectal lidocaine treatment increases coherence in the default mode network and decreases perceived pain [61], further supporting the current results on how inherent differences and associations with behavioral data can predict IBS symptom trajectory.

There were differences in signatures between short-term vs. long-term predictions. Pertinent discriminations for classifying 3-month improvers were aspects of CDRISC defined resilience. Scores for the CDRISC Persistence subscale were higher in patients without improvement and associated with lower anatomical connectivity within the dIPFC, as well as greater resting-state functional connectivity in circuits responsible for transmitting topdown spatial attention information to the visual cortex during sustained attention [62]. Further, over-engagement of attentional systems and lower inhibitory control from the dIPFC points to more severe traumatic and stressful life histories, as indicated by resilience subscale scores in non-improvers [63, 64]. Mounting evidence has shown the dIPFC acts as an interface between cognitive processing and pain regulation, shows a loss of neuronal tissue with chronic pain which is at least partially reversible [65-67]. This identifies the dIPFC as a potential therapeutic target in IBS, using transcranial magnetic stimulation, transcranial directcurrent stimulation and mindfulness, all of which have shown promise in other chronic pain populations [65].

Conversely, patients with improvements had greater scores on the CDRISC Adaptability subscale, which was associated with greater default mode resting-state connectivity and corticospinal tract integrity. The hyperconnectivity of these pathways originating in the motor cortex may constitute strengths in active top-down pain modulation. As seen in the current results, stimulation from the motor cortex can result in top-down activation via the thalamus and basal ganglia, can result in activation of the periaqueductal gray (PAG) for pain modulation, which may be a modulatory mechanism pain-relieving in the current study that requires further investigation [66]. Stimulating the motor areas in transcranial direct current stimulation has also been found to reduce pain [67]. This suggests that the brain in improvers was more "adaptative", and was better able to minimize attention to IBS symptoms and more likely to engage in top-down pain modulation via pyramidal tracts.

Pertinent behavioral variables discriminating long-term improvers included pain threshold and tolerance unpleasantness ratings from sensory testing. Greater unpleasantness ratings were associated with greater anatomical connectivity between the anterior portion of the subcentral gyrus (area 43) and posterior bank of the postcentral sulcus (area Pft)-two regions that are known to be distinctly connected via white matter projections, connected to the somatosensory strip, and play a large role in processing somatosensory information [68]. Greater unpleasantness ratings were also associated with greater S1-SMA anatomical connectivity. All pertinent features discriminating long-term improvers were associated with greater resting-state connectivity between the thalamus and superior parietal cortex, and with greater volume in the posterior insula $(\mathrm{SMN})$, a brain region receiving afferent inputs from the viscera, referred to as viscerosensory cortex [69]. This finding supports the hypothesis that circuitry in sensorimotor systems is more robust, connected 
and active in IBS patients with persistent or worsening symptom trajectories. Pain has historically been considered a bidimensional experience consisting of the sensory-discriminative dimension (often referred as "intensity"), and the affective-motivational (or "unpleasantness") dimension [70, 71]. The ascending and descending pain modulation pathways underlying the differences between pain intensity and pain unpleasantness have been well explored, showing that pain intensity is the primary contributor to pain unpleasantness and not vice-versa [72]. Our results consisted of regions known to interact to produce pain intensity, unpleasantness and secondary pain affect $[72,73]$. The anterior cingulate cortex (ACC) is known to play a large role in the modulation of unpleasantness $[72,73]$ but was not identified in the signature by DIABLO. We posit that as the ACC is involved in encoding immediate pain unpleasantness, and our baseline imaging was not conducted in conjunction with an acute pain stimulus, our protocol instead identified brain structures and pathways that are generally responsive to sensations, arousal, autonomic, somatomotor activation, and perceived threat which underlie pain unpleasantness rather than the immediate threat of the pain stimulus [72].

Study outcomes also found synergistic effects in brain regions known to be affected by early adverse life events. The greater resting-state functional connectivity between the PCC and fusiform face cortex and its positive association with early life trauma is supported by past research. The fusiform face cortex is structurally connected to the inferior longitudinal fasciculus (ILF), which is known as the visual-limbic pathway [74], and trauma such as domestic violence is associated with decreased integrity of the ILF in the developing brain $[75,76]$. Regions connected to the ILF, such as the superior temporal gyrus also had lower volume [67]. Nonimprovers also had lower cortical thickness in the second visual area (V2) - also shown to have lower integrity in the abused developing brain [74]-and lower anatomical connectivity between the V2 and hippocampus, which are connected via the ILF. This pattern was accentuated with a greater lifetime history of symptoms that accompany chronic pain, and the ILF is known to have decreased integrity associated with greater symptom severity in other chronic pain populations and the transition to chronic pain $[24,76]$. Regions at the anterior end of the ILF such as the hippocampus have been shown to play a key role in the transition from acute to chronic pain [77]. Additionally, brain regions along the ILF that are known to be associated with early-life adversity such as the hippocampus, fusiform face cortex, and visual cortex were also identified in the multi-modal signature. This indicates that neuroplastic changes in the ILF due to early life adversity may be involved in the exacerbation of IBS. For example, areas such as the hippocampus and immediate surrounding regions are known to play a crucial role in developing chronic pain, as hippocampal reorganization is associated with the transition from acute to chronic pain via abnormal learning and emotional processes [77]. Specifically in IBS, abnormal hippocampal glutamatergic transmission [78] and activation of the hippocampus in response to rectal distention [79] have also been observed. Taken together, neuroplastic changes in the ILF and connected brain regions may accentuate the risk for exacerbating IBS symptoms, highlighting the need for preventative intervention in early life when the first symptoms indicative of chronic pain development can be detected.

\section{Limitations and advantages}

The relatively small sample size should be noted as a limitation for the current study; this was due in part to participant attrition at follow-up. Furthermore, because IBS is a life-long illness for some, the study's 12-month follow-up provides only a snapshot of each patients' symptom trajectory. The literature provides evidence that early adverse life events, [10] are associated with increased risk of exacerbating IBS; yet, symptom trends over 12 months, as indicated by participants' self-reports, does not establish causation. A comparison of healthy volunteers or another visceral pain control such as inflammatory bowel disease or interstitial cystitis (IC)/bladder pain syndrome (BPS) would provide further insight into pain-related, brain mechanistic pathways [80]. Functional life impairment, and the impact of daily life due to symptoms were not assessed but would be important in future studies and analyses [20]. Additionally, as this is a difficult population sample to recruit, the time-of-day measures that were assessed can vary from $8 \mathrm{AM}$ to $6 \mathrm{PM}$. As circadian rhythms can be seen in virtually every physiological process in the body, including the central nervous system, and seen in many brain-related disorders [81, 82], future studies should consider integrating circadian rhythms into the study design and analysis. As one would expect stress, anxiety and depression to be pertinent variables of interest, the reason these likely were not identified by DIABLO is the participants recruited did not have and diagnosable psychiatric conditions. This would result in a limited range of mood variables. Additionally, the short nature of the follow-up in this long-term illness is likely leading to the identification of specific types of predictors. Future studies should also monitor hormonal changes at different phases in the menstrual cycle at the time of testing. Menstrual cycle status can be estimated in our sample by calculating the number of days between the first day of their menstrual period and the day of scan (nine women were in the menstrual phase, 13 in the follicular phase, and 27 in the luteal phase of the data available), but without ovulation kits, exact status is unknown. Strengths of the study included measuring symptom changes longitudinally over 12 months, as opposed to a cross-sectional study. This can also be further strengthened by including multi-modal brain imaging at multiple time points, along with other potentially related biomarkers from areas such as the microbiome, genetics and the immune system. This is also the first study to use multi-modal brain imaging and clinical data in an integrative manner to predict symptom trajectory in a sample of patients with IBS.

\section{CODE AVAILABILITY}

All analyses after data processing were done using the mixOmics package [47] version 6.17.27 in $\mathrm{R}$ version 4.1.0. Code and data are available on request.

\section{REFERENCES}

1. Enck P, Aziz Q, Barbara G, Farmer AD, Fukudo S, Mayer EA, et al. Irritable bowel syndrome. Nat Rev Dis Prim. 2016;2:404-13.

2. Lovell RM, Ford AC. Global prevalence of and risk factors for irritable bowel syndrome: a meta-analysis. Clin Gastroenterol Hepatol. 2012;10:712-21.

3. Chey WD, Kurlander J, Eswaran S. Irritable bowel syndrome: a clinical review. JAMA. 2015;313:949-58.

4. Gralnek IM, Hays RD, Kilbourne AA, Naliboff B, Mayer EA. The impact of irritable bowel syndrome on health-related quality of life. Gastroenterology 2000;119:654-60.

5. Khan S, Chang L. Diagnosis and management of IBS. Nat Rev Gastroenterol Hepatol. 2010;7:565-81.

6. Niculescu AB, Le-Niculescu H, Levey DF, Roseberry K, Soe KC, Rogers J, et al. Towards precision medicine for pain: diagnostic biomarkers and repurposed drugs. Mol Psychiatry. 2019;24:501-22.

7. Wang XJ, Camilleri M. Personalized medicine in functional gastrointestinal disorders: understanding pathogenesis to increase diagnostic and treatment efficacy. World J Gastroenterol. 2019;25:1185-96.

8. Vardeh D, Mannion RJ, Woolf CJ. Toward a mechanism-based approach to pain diagnosis. J Pain 2016;17:T50-T69.

9. Mayer EA, Gupta A, Kilpatrick LA, Hong J-Y. Brain mechanisms in chronic visceral pain. Pain 2015;156:S50-63.

10. Mayer EA, Labus JS, Tillisch K, Cole SW, Baldi P. Towards a systems view of IBS. Nat Rev Gastroenterol Hepatol. 2015;12:592-605.

11. Mayer EA. Gut feelings: the emerging biology of gut-brain communication. Nat Rev Neurosci. 2011;12:453-66.

12. Mayer EA, Labus J, Aziz Q, Tracey I, Kilpatrick L, Elsenbruch S, et al. Role of brain imaging in disorders of brain-gut interaction: a Rome Working Team Report. Gut 2019;68:1701-15. 
13. Bradford K, Shih W, Videlock EJ, Presson AP, Naliboff BD, Mayer EA, et al. Association between early adverse life events and irritable bowel syndrome. Clin Gastroenterol Hepatol. 2012;10:385-90.

14. Gupta A, Kilpatrick L, Labus J, Tillisch K, Braun A, Hong J-Y, et al. Early adverse life events and resting state neural networks in patients with chronic abdominal pain: evidence for sex differences. Psychosom Med. 2014;76:404-12.

15. Liu S, Hagiwara SI, Bhargava A. Early-life adversity, epigenetics, and visceral hypersensitivity. Neurogastroenterol Motil. 2017;29:e13170.

16. Farnam A, Somi MH, Sarami F, Farhang S. Five personality dimensions in patients with irritable bowel syndrome. Neuropsychiatr Dis Treat. 2008;4:959-62.

17. Tkalčić $M$, Hauser $G$, Štimac D. Differences in the health-related quality of life, affective status, and personality between irritable bowel syndrome and inflammatory bowel disease patients. Eur J Gastroenterol Hepatol. 2010;22:862-7.

18. Lackner JM, Gurtman MB. Pain catastrophizing and interpersonal problems: a circumplex analysis of the communal coping model. Pain 2004;110:597-604.

19. Drossman DA. Do psychosocial factors define symptom severity and patient status in irritable bowel syndrome? Am J Med. 1999;107:41-50.

20. Addante R, Naliboff B, Shih W, Presson AP, Tillisch K, Mayer EA, et al. Predictors of health-related quality of life in irritable bowel syndrome patients compared with healthy individuals. J Clin Gastroenterol. 2019;53:E142-E149.

21. Surdea-Blaga T, Băban A, Dumitrascu DL. Psychosocial determinants of irritable bowel syndrome. World J Gastroenterol. 2012;18:616-26.

22. Kutch JJ, Labus JS, Harris RE, Martucci KT, Farmer MA, Fenske S, et al. Resting-state functional connectivity predicts longitudinal pain symptom change in urologic chronic pelvic pain syndrome. Pain 2017;158:1.

23. Baliki MN, Petre B, Torbey S, Herrmann KM, Huang L, Schnitzer TJ, et al. Corticostriatal functional connectivity predicts transition to chronic back pain. Nat Neurosci. 2012;15:1117-9.

24. Mansour A, Baliki MN, Huang L, Torbey S, Herrmann K, Schnitzer TJ, et al. Brain white matter structural properties predict transition to chronic pain. Pain 2013;154:2160-8.

25. Vachon-Presseau E, Té P, Petre B, Huang L, Berger SE, Torbey S, et al. Corticolimbic anatomical characteristics predetermine risk for chronic pain. Brain 2016;139:1958-70.

26. Singh A, Shannon CP, Gautier B, Rohart F, Vacher M, Tebbutt SJ, et al. DIABLO: an integrative approach for identifying key molecular drivers from multi-omics assays. Bioinformatics 2019;35:3055-62.

27. Drossman DA. The functional gastrointestinal disorders and the Rome III process. Gastroenterology 2006;130:1377-90.

28. Francis CY, Morris J, Whorwell PJ. The irritable bowel severity scoring system: a simple method of monitoring irritable bowel syndrome and its progress. Aliment. Pharmacol Ther. 1997;11:395-402.

29. Lovell RM, Ford AC. Effect of gender on prevalence of irritable bowel syndrome in the community: systematic review and meta-analysis. Am J Gastroenterol. 2012;107:991-1000.

30. Talley NJ, Newman P, Boyce PM, Paterson KJ, Owen BK. Initial validation of a bowel symptom questionnaire and measurement of chronic gastrointestinal symptoms in Australians. Aust NZ J Med. 1995;25:302-8.

31. Zigmond AS, Snaith RP. The Hospital Anxiety and Depression Scale. Acta Psychiatr Scand. 1983;67:361-70.

32. Spielberger CD. State-trait anxiety inventory (STAI). Mind Gard. 1983;94061: 261-3500.

33. Cohen S, Kamarck T, Mermelstein R. A global measure of perceived stress. J Health Soc Behav. 1983;24:385-96.

34. Goldberg LR, Johnson JA, Eber HW, Hogan R, Ashton MC, Cloninger CR, et al. The international personality item pool and the future of public-domain personality measures. J Res Personal. 2006:40:84-96.

35. Bremner JD, Bolus R, Mayer EA. Psychometric properties of the early trauma inventory-self report. J Nerv Ment Dis. 2007;195:211-8.

36. Williams DA, Schilling S. Advances in the assessment of fibromyalgia. Rheum Dis Clin N Am. 2009;35:339-57.

37. Connor KM, Davidson JRT. Development of a new Resilience scale: the ConnorDavidson Resilience scale (CD-RISC). Depress Anxiety 2003;18:76-82.

38. Zhou Q, Fillingim RB, lii JLR, Verne GN, States U. Thermal hypersensitivity in a subset of irritable bowel syndrome patients. World J Gastroenterol. 2009;15:3254-60.

39. Gracely RH, McGrath F, Dubner R. Ratio scales of sensory and affective verbal pain descriptors. Pain 1978;5:5-18.

40. Glasser MF, Coalson TS, Robinson EC, Hacker CD, Harwell J, Yacoub E, et al. A multi-modal parcellation of human cerebral cortex. Nature 2016;536:171-8.

41. Arslan S, Ktena SI, Makropoulos A, Robinson EC, Rueckert D, Parisot S. Human brain mapping: a systematic comparison of parcellation methods for the human cerebral cortex. Neurolmage 2018;170:5-30.

42. Desikan RS, Ségonne F, Fischl B, Quinn BT, Dickerson BC, Blacker D, et al. An automated labeling system for subdividing the human cerebral cortex on MRI scans into gyral based regions of interest. Neurolmage 2006;31:968-80.
43. Makris N, Goldstein JM, Kennedy D, Hodge SM, Caviness VS, Faraone SV, et al. Decreased volume of left and total anterior insular lobule in schizophrenia. Schizophr Res. 2006;83:155-71

44. Frazier JA, Chiu S, Breeze JL, Nikos Makris M, Lange N, David Kennedy SN, et al. Structural brain magnetic resonance imaging of limbic and thalamic volumes in pediatric bipolar disorder. Am J Psychiatry. 2005;162:1256-65.

45. Goldstein JM, Seidman $\amalg$, Makris N, Ahern T, O'brien LM, Caviness VS, et al. Hypothalamic abnormalities in schizophrenia: sex effects and genetic vulnerability. Biol Psychiatry. 2007;61:935-45.

46. Tenenhaus A, Philippe C, Guillemot V, Le Cao KA, Grill J, Frouin V. Variable selection for generalized canonical correlation analysis. Biostatistics 2014;15:569-83.

47. Rohart F, Gautier B, Singh A, Lê Cao KA. mixOmics: an R package for 'omics feature selection and multiple data integration. PLoS Comput Biol 2017;13:1-19.

48. González I, Cao KAL, Davis MJ, Déjean S. Visualising associations between paired "omics" data sets. BioData Min. 2012;5:1-23.

49. Hosmer DW, Lemeshow S, Sturdivant RX. Applied logistic regression. 3rd edn. Hoboken:Wiley;2013.

50. Kucyi A, Davis KD. The neural code for pain: from single-cell electrophysiology to the dynamic pain connectome. Neuroscientist. 2016;23:1-18.

51. Schooler JW, Smallwood J, Christoff K, Handy TC, Reichle ED, Sayette MA. Metaawareness, perceptual decoupling and the wandering mind. Trends Cogn Sci. 2011;15:319-26.

52. Kucyi A, Davis KD. The dynamic pain connectome. Trends Neurosci. 2015;38: 86-95.

53. Andrews-Hanna JR, Smallwood J, Spreng RN. The default network and selfgenerated thought: component processes, dynamic control, and clinical relevance. Ann N Y Acad Sci 2014;1316:29-52.

54. Naliboff BD, Smith SR, Serpa JG, Laird KT, Stains J, Connolly LS, et al. Mindfulnessbased stress reduction improves irritable bowel syndrome (IBS) symptoms via specific aspects of mindfulness. Neurogastroenterol Motil 2020;32:e13828.

55. Kucyi A, Salomons TV, Davis KD. Mind wandering away from pain dynamically engages antinociceptive and default mode brain networks. PNAS 2013;110:18692-7.

56. Labus JS, Hollister EB, Jacobs J, Kirbach K, Oezguen N, Gupta A, et al. Differences in gut microbial composition correlate with regional brain volumes in irritable bowel syndrome. Microbiome. 2017;5:1-17.

57. Videlock EJ, Adeyemo $M$, Licudine A, Hirano M, Ohning G, Mayer $M$, et al. Childhood trauma is associated with hypothalamic-pituitary-adrenal axis responsiveness in irritable bowel syndrome. Gastroenterology 2009;137:1954-62.

58. Sansone RA, Sansone LA. Irritable bowel syndrome: relationships with abuse in childhood. Innov Clin Neurosci. 2015;12:34-7.

59. Bhatt RR, Gupta A, Labus JS, Zeltzer LK, Tsao JC, Shulman RJ, et al. Altered brain structure and functional connectivity and its relation to pain perception in girls with irritable bowel syndrome. Psychosom Med. 2019;81:146-54.

60. Bhatt RR, Gupta A, Mayer EA, Zeltzer LK. Chronic pain in children: structural and resting-state functional brain imaging within a developmental perspective. Pediatr Res. 2019;88:1-10.

61. Letzen JE, Craggs JG, Perlstein WM, Price DD, Robinson ME. Functional connectivity of the default mode network and its association with pain networks in irritable bowel patients assessed via lidocaine treatment. J Pain. 2013;14:1077-87.

62. Lauritzen TZ, D'Esposito M, Heeger DJ, Silver MA. Top-down flow of visual spatial attention signals from parietal to occipital cortex. J Vis. 2009;9:18-18.

63. Scali J, Gandubert C, Ritchie K, Soulier M, Ancelin ML, Chaudieu I. Measuring resilience in adult women using the 10-items Connor-Davidson resilience scale (CDRISC). Role of trauma exposure and anxiety disorders. PLoS ONE. 2012;7:e39879.

64. Wu G, Feder A, Cohen H, Kim JJ, Calderon S, Charney DS, et al. Understanding resilience. Front Behav Neurosci. 2013;7:10.

65. Seminowicz DA, Moayedi M. The dorsolateral prefrontal cortex in acute and chronic pain. J Pain. 2017;18:1027-35.

66. Garcia-Larrea L, Peyron R. Motor cortex stimulation for neuropathic pain: from phenomenology to mechanisms. Neurolmage 2007;37:71-9.

67. O'Connell NE, Marston L, Spencer S, Desouza LH, Wand BM. Non-invasive brain stimulation techniques for chronic pain. Cochrane Datab Syst Rev. 2018;3: CD008208.

68. Baker CM, Burks JD, Briggs RG, Conner AK, Glenn CA, Robbins JM, et al. A connectomic Atlas of the human cerebrum-chapter 5: the insula and opercular cortex. Oper Neurosurg 2018;15:S175-S244.

69. Gehrlach DA, Dolensek N, Klein AS, Roy Chowdhury R, Matthys A, Junghänel M, et al. Aversive state processing in the posterior insular cortex. Nat Neurosci. 2019;22:1424-37.

70. Auvray M, Myin E, Spence C. The sensory-discriminative and affectivemotivational aspects of pain. Neurosci Biobehav Rev. 2010;34:214-23.

71. Talbot K, Madden VJ, Jones SL, Moseley GL. The sensory and affective components of pain: are they differentially modifiable dimensions or inseparable aspects of a unitary experience? A systematic review. Br J Anaesth. 2019;123:e263-e272. 
72. Price DD. Psychological and neural mechanisms of the affective dimension of pain. Science 2000;288:1769-72.

73. Bushnell MC, Čeko M, Low LA. Cognitive and emotional control of pain and its disruption in chronic pain. Nat Rev Neurosci. 2013;14:502-11.

74. Baker CM, Burks JD, Briggs RG, Stafford J, Conner AK, Glenn CA, et al. A connectomic Atlas of the human cerebrum-chapter 9: the occipital lobe. Oper Neurosurg 2018;15:S372-S406.

75. Teicher MH, Samson JA, Anderson CM, Ohashi K. The effects of childhood maltreatment on brain structure, function and connectivity. Nat Rev Neurosci. 2016;17:652-66.

76. Choi J, Jeong B, Polcari A, Rohan ML, Teicher MH. Reduced fractional anisotropy in the visual limbic pathway of young adults witnessing domestic violence in childhood. Neurolmage 2012;59:1071-9.

77. Farmer MA, Huang L, Martucci K, Yang CC, Maravilla KR, Harris RE, et al. Brain white matter abnormalities in female interstitial cystitis/bladder pain syndrome: a MAPP network neuroimaging study. J Urol. 2015;194:118-26.

78. Mutso AA, Petre B, Huang L, Baliki MN, Torbey S, Herrmann KM, et al. Reorganization of hippocampal functional connectivity with transition to chronic back pain. J Neurophysiol. 2014;111:1065-76.

79. Niddam DM, Tsai SY, Lu CL, Ko CW, Hsieh JC. Reduced hippocampal glutamateglutamine levels in irritable bowel syndrome: preliminary findings using magnetic resonance spectroscopy. Am J Gastroenterol. 2011;106:1503-11.

80. Kwan CL, Diamant NE, Pope G, Mikula K, Mikulis DJ, Davis KD. Abnormal forebrain activity in functional bowel disorder patients with chronic pain. Neurology. 2005;65:1268-77.

81. Grundy L, Erickson A, Brierley SM. Visceral pain. Annu Rev Physiol. 2019;81:261-84

82. Logan RW, McClung CA. Rhythms of life: circadian disruption and brain disorders across the lifespan. Nat Rev Neurosci. 2019;20:49-65.

\section{ACKNOWLEDGEMENTS}

We acknowledge the invaluable editorial services by Cathy Liu, Parth R. Bhat and Cynthia L. Boyle, PhD. We would further like to acknowledge Parth R. Bhatt and his company (www.parth.pro) for helping generate the figures in this manuscript.

\section{FUNDING}

Funding was supported by the National Institute of Diabetes, Digestive and Kidney Diseases (NIDDK), grant number R01DK048351-19 awarded to Emeran Mayer, MD and by the CURE grant number P30-DK04130. Ravi R. Bhatt's graduate studies are supported by the National Science Foundation GRFP grant number 2020290241.

\section{AUTHOR CONTRIBUTIONS}

EAM was responsible for study conception, funding. JSL, EAM were responsible for collecting and supervising data collection. RRB and EAM interpreted the results. RRB conducted data analysis, wrote and revised the paper. $R R B, A G, C L$, and PPV were responsible for data processing. AG, JSL, BDN, and EAM supervised data anlaysis, wrote and revised the paper.

\section{COMPETING INTERESTS}

Emeran A. Mayer, MD is a scientific advisory board member of Danone, Axial Biotherapeutics, Viome, Amare, Mahana Therapeutics, Pendulum, Bloom Biosciences and APC Microbiome Ireland. No other authors have no conflicts of interest to disclose.

\section{ADDITIONAL INFORMATION}

Supplementary information The online version contains supplementary material available at https://doi.org/10.1038/s41380-021-01375-9.

Correspondence and requests for materials should be addressed to Emeran A. Mayer.

Reprints and permission information is available at http://www.nature.com/ reprints

Publisher's note Springer Nature remains neutral with regard to jurisdictional claims in published maps and institutional affiliations. Attribution 4.0 International License, which permits use, sharing, adaptation, distribution and reproduction in any medium or format, as long as you give appropriate credit to the original author(s) and the source, provide a link to the Creative Commons license, and indicate if changes were made. The images or other third party material in this article are included in the article's Creative Commons license, unless indicated otherwise in a credit line to the material. If material is not included in the article's Creative Commons license and your intended use is not permitted by statutory regulation or exceeds the permitted use, you will need to obtain permission directly from the copyright holder. To view a copy of this license, visit http://creativecommons. org/licenses/by/4.0/.

(c) The Author(s) 2021 